\title{
A Biophysical Approach to SARS-CoV-2 Pathogenicity
}

\author{
Claudio Messori \\ Department of Rehabilitation, U.S.L. Company Parma, Parma, Italy \\ Email: messori.claudio@gmail.com
}

How to cite this paper: Messori, C. (2021) A Biophysical Approach to SARS-CoV-2 Pathogenicity. Open Access Library Journal, 8: e8183.

https://doi.org/10.4236/oalib.1108183

Received: November 12, 2021

Accepted: December 24, 2021

Published: December 27, 2021

Copyright $\odot 2021$ by author(s) and Open Access Library Inc.

This work is licensed under the Creative Commons Attribution International License (CC BY 4.0).

http://creativecommons.org/licenses/by/4.0/

\section{(c) (i) Open Access}

\begin{abstract}
In order to infect the target host cell, a virus like severe acute respiratory syndrome coronavirus 2 (SARS-CoV-2), whose lytic/virulent lifestyle involves the infection, replication and lysis of the host cell, must be able to penetrate its membrane for the release of viral progeny. For this purpose, its action takes place both on a biophysical and biochemical level. Viral action begins with the alteration of physiological interactions held by the target cell with extracellular aqueous electrolytic solutions (membrane-ions interactions), followed by the disruption of the membrane structural properties. In the present paper the earliest virus-host biophysical events are addressed, in particular the electrostatic (short-range) and electrodynamic (long-range) interactions. In section one a biophysical profile of SARS-CoV-2 pathogenicity is outlined. The prominent electrostatic events that characterize the earliest stages of virus-host interactions are taken into account. The host cell oxidative stress reaction is discussed. In section two, biological water as biphasic liquid and structured interfacial water's fourth phase are introduced by resorting to Quantum Field Theory (QFT) and Quantum Electrodynamic (QED). Structured interfacial water as a semiconductor and the proton-based self-driven liquid-flow system are also discussed. In section three it is assumed that in its approach to the target host cell SARS-CoV-2 triggers the cellular phase-matching feature (which operates as a very selective filter discriminating among perturbations and stimuli that are out of phase with the oscillatory motions allowed by the cell's inner dynamics) by emitting a stressful ultra low frequency-electromagnetic field (ULF-EMF), which generates an alert response within the cell by influencing ionic fluxes throughout cellular membrane. Therefore, it is suggested that the earliest virus-host interaction would rest on electromagnetic long-range events, before electrostatic and chemical interactions occur, which are influencing the redox potential of the target host-cell's membrane chemical species, affecting its acid-base equilibria, therefore inducing a cellular stress response (e.g. via plas-
\end{abstract}


ma-membrane-localized redox signalling) with reactive oxygen species (ROS) and reactive nitrogen species (RNS) production. Accordingly, redox-responsive intracellular signaling and anti-inflammatory scavenging systems would be remotely (pre) activated by non-ionizing interference phenomena, before virus-cell come together, followed by electrostatic and chemical events that provoke a branching, cascade-like, chain of reactions. A better understanding of the earliest of biophysical events will enable a more rational approach to dealing with both the binding of the SARS-CoV-2 highly glycosylated Spike's S1 subunit receptor binding domain (RBD) to the host cell's angiotensin-converting enzyme 2 (ACE2) and the direct interaction with the lipid bilayer on the cell surface.

\section{Subject Areas}

Infectious Diseases

\section{Keywords}

Non-Ionizing Interference Phenomena, Electromagnetotaxis, Ion Channelling Activity, Electron-Proton Transductions and Exchange, Redox Balance, Oxidative Stress Reactions, Structured Interfacial Water CD/EZ; Interfacial Water Stressors

\section{Introduction}

Viruses have been found everywhere on Earth. Researchers estimate that viruses outnumber bacteria by 10 to 1 . They are strongly dependent on the host of one or the other of the three primary kingdoms, namely archaea, prokaryotes and eukaryotes, and must exploit and overcome membrane barriers, at first, to infect cells, i.e. hijacking the host cell machinery to help with entry replication, packaging and release of progeny to infect new cells. Often, they kill the host cell in the process, and cause damage to the host organism.

To their purpose, viruses with a lytic/virulent lifestyle must first be able to evade the host antiviral immune activation, as to intercept the target cell, engage it and promote viral infection by disrupting the cellular environment and therefore the membrane integrity. To perform these initial steps of viral infection, viruses follow two intertwined paths, a biochemical and a biophysical one.

Nowadays, the immune system and cellular stress response is thought to be activated by "danger signals" or perturbing agents, such as high and low temperature, changes in $\mathrm{pH}$, toxic metals and compounds, ionizing radiation (thermal effect) and pathogens like viruses and bacteria, which are relevant to the induction of inflammation and immune responses.

Biological systems are currently conceived as a set of substantially isolated molecules, mechanical-like systems consisting of independent, incoherent molecules, subjected to disturbing agents and the driving effect of an assortment of 
external bias (mixotaxis ${ }^{1}$ ).

The production, emission and absorption of non-ionizing electromagnetic fields by biological systems, as well as their role in biological dynamics and in organism-organism organism-environment interactions are widely demonstrated [3]-[8], but non-ionizing radiation is still not considered relevant to the induction of inflammation and immune responses.

The forces of attraction are largely electrostatic in nature and vary with distance as the interaction between dipoles is shielded by the dielectric constant of the medium. Like other dipoles, water molecules can stack together in dipole interactions with alternating positive and negative poles next to each other. It can also engage in electrostatic interactions with charged ions and other dipoles dissolved in it. It is primarily the charge distribution on the surface of molecules that determines their physical and chemical properties, such as bonding ability, orientation, and mutual position. When the geometrical configuration of two molecules fits in such a way that a minimum of the (electrical) Coulomb potentials of the valence electrons is achieved, a chemical bonding can take place. However, charge distribution through its time variations is also responsible for the properties of the electromagnetic (EM) radiation that molecules emit, such as polarization, spatial distribution, and direction, and for their interaction with impinging radiation. The interaction of radiation with matter is only possible through redistribution of charge. Structural changes in the molecules entail charge shifts and thus changes in the electromagnetic field (EMF) envelope of the molecule, which may be the real mediator of the molecule's interaction with other molecules [9].

Biological systems have high water content containing ions. Ions in water are not just simple charged particles as one would expect to observe in a vacuum, as the charges attract molecules of water that may be bound to them in a variety of configurations and with bonds of varying strength [10].

Ion-specific effects are widespread, but nowhere are more critically manifested than at the fluid interfaces of biological structures. Action potentials, osmotic flows, energy transduction, and the stabilization of proteins are driven by ion concentration gradients across liquid films on hydrophobic biomaterials. Experiments revealed that ions interact specifically at the prototype air-water interface over separations that vastly exceed the range of direct electrostatic forces in any dielectric medium [11]. Such long-range specific ion effects may be triggered by electrostatic and electrodynamic forces, but they must be powered also by other mechanisms, such as the thermal fluctuations intrinsic to fluid interfaces.

Chemical bonds (including covalent, ionic, hydrogen and van der Waals types) have been commonly assumed to be dominating for biological organiza-

${ }^{1}$ Directed cell interaction both in vitro and in vivo has been shown to be driven by the guiding effect of local and non-local anisotropic features in the cell environment, i.e. by an assortment of external biasing cues, the so called mixotaxis, ranging from gradients of soluble (chemotaxis) to bound (haptotaxis) molecules, gradients of mechanical properties (durotaxis), electric fields (electrotaxis) as well as iterative biases in the environment topology (ratchetaxis) [1] [2]. 
tion and activity. However, these bonds represent forces acting at short distances in the $\mathrm{nm}$ region. Biological systems maintain coherence at every dimension scale. Long-range coherence, large distance cooperation, and the whole body control are significant properties of biological systems. Short range interaction, if it is there, is made possible by the long-range one which brings "near" the components (e.g., making possible the formation of hydrogen-bonds in water).

EMFs generated in biosystems support such long-range coherence and is a nature of Earth's biological phenomenon, from viruses [12] to archaea, prokaryotic [13] and eukaryotic cells to pluricellular organisms [14] and human beings.

Hydrogen-bonds (HBs) in cells (likely also in virions) act as piezoelectric centers of energy transduction with biophysical characteristics, and can be considered to be electromagnetic (EM) radiation emission centers. The internal regions of proteins favor the creation of $\mathrm{HB}$ networks producing in the extracellular matrix and cytoskeletal system (including actin and ATP molecules) proton semi-conduction systems. The $\mathrm{HB}$ networks act as proton conductors and are responsible for photon-conformational interactions able to do biophysical work. According to [15], the passage of a proton along channels of $\mathrm{HB}$ ( $\alpha$-helices and $\beta$-sheets) can alter the frequency of the EM radiation emitted by the HBs they contain, by which protonic conformational interactions may signal the change in the protein conformation at a distance and in real time, as well as performing the mechanical work (such as adaptation between enzyme and substratum).

In ordered biosystems, because of the very large number of HBs in the cell, EM emission from a complex harp orchestra takes place uniformly and in all directions. In several diseases, including COVID-19, uniformity and unidirectionality is lost and the system breaks in chaotic, heterogeneous models which use more energy $(\Delta \mathrm{E})$ from the normal structures taxing their abilities to continue in performance [16].

All standard chemistry is totally reliant on electrostatics and avoids all mention of electrodynamics and the consequent radiation field, which is supporting the notion of water as a primary mediator of biological effects induced via electromagnetic means into living systems. The electrodynamic structure of interfacial water Coherence Domainel Exclusion Zone (CD/EZ) (see Section 2) can be regarded as the main agents of the self-organization of living organisms. Given the basically electromagnetic character and piezoelectric properties of this organization, it is not surprising that biological systems interact with endogenous/exogenous sonic signals (phonons) and with ultra low frequency-electromagnetic field (ULF-EMF) in a non-ionizing way.

The role played in structured interfacial water $C D / E Z$ by sonic signals (phonons) and non-ionizing EM radiation (photons) ${ }^{2}$ emitted and absorbed by biological systems, which do not cause thermal or heating effects but do cause

${ }^{2}$ Coherent mechanical vibrations (phonons) of living cells are measured by atomic force microscopy in the acoustic frequency range. Frequencies of the mechanical vibrations and of the electromagnetic field (photons) generated by a cell are equal. 
non-thermal biological effects on electron and proton transfer reactions, enzymes, DNA and fluxes in the ion channels of excitable membranes, is currently ignored or neglected.

Likely, among the biophysical modalities involved in the pathogenicity of SARS-CoV-2 (severe acute respiratory syndrome coronavirus 2 ) and in addition to the classical electrostatic modality, other modalities based sonic signaling and on ULF-EMF signaling [17] should be taken into account. The present work points at introducing the possible ULF-EMF interactions.

\section{SARS-CoV-2 the Virus}

SARS-CoV-2 (severe acute respiratory syndrome coronavirus 2), the causative agent of the coronavirus disease 2019 (COVID-19) pandemic (whose immunopathogenesis remains at date poorly defined), belongs to the virus order Nidovirales, family Coronaviridae, genus Betacoronavirus (enveloped, positive-sense, single-stranded RNA viruses that are zoonotic) and sub-genus Sarbecovirus, same as SARS-COV-1 the aetiologic agent of the SARS outbreak emerged in the Guangdong province in China in 2002, with likely origin in bats, and likely involvement of another (intermediate) host animal such as the pangolin.

The most distinctive feature of Coronaviridae family is genome size: coronaviruses have the largest genomes among all RNA viruses, including those RNA viruses with segmented genomes. This expansive coding capacity seems to both provide and necessitate a wealth of gene-expression strategies, most of which are incompletely understood [18].

SARS-CoV-2 evolutionary path to humans has not been defined, and remains controversial. Possibilities include direct spillover from a bat to humans, indirect spillover from a bat to humans through an intermediate host (as for SARS and MERS), and a leak from a laboratory studying bat coronaviruses, either from bona fide SARS-CoV-2 isolated from a natural sample or from a derivative of a natural virus generated by serial passage in laboratory animals and/or cell culture. At present, there is no direct evidence that SARS-CoV-2 was present in any laboratory before it was first discovered in a patient, and detailed sequence analyses have been performed to argue that it most likely spilled over naturally [19].

The closest known related coronavirus sequence, designated RaTG13 (96.2\% identical genome-wide to SARS-CoV-2), was identified by scientists from the Wuhan Institute of Virology (WIV) in a bat fecal sample isolated from a cave in Yunnan, China, in 2013 [20]. The complete sequence of RaTG13 was disclosed at the same time as the sequence of SARS-CoV-2 by the same WIV research group, which has been studying emerging infectious diseases and coronaviruses, particularly bat coronaviruses, for about 20 years. The $3.8 \%$ nucleotide position differences separating RaTG13 and SARS-CoV-2 are estimated to represent decades in evolutionary distance in nature, although this gap could potentially be breached much more rapidly by serial passage in a laboratory, for which there is currently no direct evidence. Sequence analysis has suggested that any effort to 
derive SARS-CoV-2 by serial passage of RaTG13 or another related coronavirus would have had to occur in vivo since there is sequence evidence of conserved glycosylation sites in the $\mathrm{S}$ protein receptor binding domain indicating that it most likely evolved under immune pressure [19].

Coronaviruses constitute a special category of viruses whose genome is hosted into protein cells, with rod-shaped spikes projecting from their surfaces [21].

The name coronavirus it has been coined by June D. Almeida in 1968, who visualized (1967) the virus by Electron Microscopy (EM) [22] and derived the name from the characteristic morphology in negative-stained electron microscopy, marked by a "fringe" of surface structures described as "spikes" or "club-like" projections, that give the viral particles the appearance of having the corona of the sun seen during an eclipse (virologists compared "the characteristic 'fringe' of projections" on the outside of the virus with the solar corona, and not, as some have suggested, with the points on a crown [23]) (Figure 1).

In electron micrographs, coronavirus seems similar to a distinct pair of electron-dense shells, with a small central electron-dense area or "dimple" [24] [25] surrounded by an electron-dense ionized plasma, i.e. a corona shaped electrostatic discharge surrounding the spikes sharp particles "fringe" projections, which may interfere with the target cell's plasma-membrane-localized redox signalling [26] causing dysregulation and possibly disruption of normal cell physiology.

\subsection{SARS-CoV-2 Structural Features}

SARS-CoV-2 is a quasi-spherical-shaped enveloped virion positively charged (total structural electric charge of SARS-CoV-2 is positive, depending on the cumulative charges of its genetic material and that of the surface proteins [27] [28]) with a single positive polarity RNA strand. Its RNA genome is similar to that of other coronaviruses and has four genes that encode as many structural
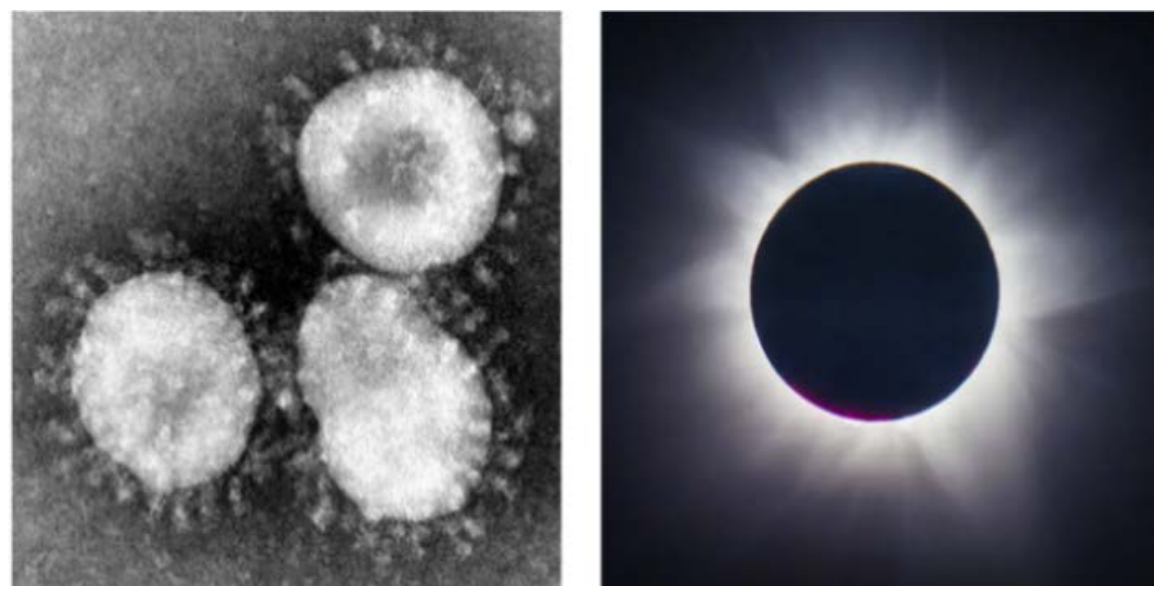

Figure 1. Left: The virions of coronaviruses (when seen by negative stain electron microscopy). Right: The corona of the sun seen during an eclipse (resembling the virions of coronaviruses when seen by thin section electron microscopy). Image source: Aronson, J. K. (2020) Coronaviruses-a general introduction, Centre for Evidence-Based Medicine https://www.cebm.net/covid-19/coronaviruses-a-general-introduction/. 
transmembrane proteins, they are: the nucleocapsid phosphoprotein $(\mathrm{N})$, which encapsulates the RNA strand forming the helical filamentous ribonucleoprotein (RNP) complex, the envelope (E) protein, the matrix or membrane (M) protein and the spike protein (S) or peplomer or S-protein, the heavily glycosylated protein that decorates the surface of coronavirus by forming characteristic spikes sharp particles (spikes are very rarely as clear in thin-sectioned EM specimens as they are when seen by negative stain EM), fundamental for the virus to enter the host cell. The E, M and S proteins are embedded in the viral quasi-fluid-like, pleomorphic, bilipid envelope, and together comprise the virus interface to the external environment. Another envelope protein, hemagglutinin-esterase (HE), serves as a receptor-destroying enzyme. HE and $S$ are functionally intertwined, binding of receptor by $S$ and destruction of receptor by HE need to be carefully balanced for efficient (pre) attachment.

The envelope E, membrane M proteins and helical nucleocapsid of enveloped viruses are morphologic features of these pathogens that confer protection to them outside their host cells. The helical nucleocapsid is visible in coronaviruses in thin section EM, which can be seen in cross sections as electron-dense black dots $6-12 \mathrm{~nm}$ in diameter on the inside of the viral particles [23]. The lack of these dots in a subcellular structure is a good indicator that it is not coronavirus.

However, the lipid bilayer within the cell membrane of enveloped viruses consists of cholesterols and phospholipids, and will only allow the virus to survive for a limited time outside the host cell environment. Hence, to survive, also enveloped viruses need to be transferred directly from one host to another, as quickly as possible.

Despite their complexity and range of function however, the structural proteins occupy only about a third of the coding capacity of the genome. A much larger section of the genome, some two-thirds, encode the nonstructural proteins (NSP) of the virus, which are required at various stages of the virus replication cycle. SARS-CoV-2 may require an acidic environment for its entry and for its ability to bud and infect bystander cells [29]. SARS-CoV-2 main protease 3CL $\mathrm{M}^{\text {pro }}$ is indispensable for virus replication and do affect the host cell protonation state by increasing its acidity degree $\left(\mathrm{pH}<\mathrm{pK}_{\mathrm{a}}\right)$ [30] [31].

The most pronounced difference between SARS-CoV-1 spike proteins and SARS-Cov-2 spike proteins is an additional (polybasic) furin cleavage site cleaved by furin-like proteases, which is essential for efficient viral entry into human lung cells, especially in cell-cell fusion to form syncytium to facilitate viral spread from one cell to another. Different cleavage sites targeted by different proteases are often associated with drastically different virulence and host cell tropism in various RNA viruses. Unlike other proteases, e.g. trypsin-like proteases, furin-like proteases are ubiquitous in human tissues. This means that if the virus gains a furin cleavage site, cellular entry has no restrictions, resulting in dramatic broadening of host cell tropism. In this context, the S-protein contributes to host specificity, and also to tissue specificity through its differential requirement of tissue-specific proteases. For this reason, viruses with different 
cell tropism may accumulate tissue-specific genomic signatures [32].

Another significant difference between SARS-CoV-1 and SARS-CoV-2 concerns the structure of two prominent viroporins, the Orf3a and Envelope (E) protein [33], of the three viroporins (the other one being Orf8a) encoded both by SARS-CoV-1 and SARS-CoV-2 (while many coronaviruses encode two viroporins, SARS-CoV encodes three [34]).

Viroporins form multimeric complexes that act as ion channels and are important players of the viral life cycle of SARS-CoV-2 and one of the primary determinants of its pathogenesis, promoting ion imbalances within cells [35] and/or disrupting cellular pathways through protein-protein interaction [36] [37] [38], acting as interfacial water stressors, implicated both in host cell apoptosis and proinflammatory cytokines storm.

Sarkar and colleagues in [33] found that Orf3a has several hotspots of mutations which has been reported in SARS-CoV-2 with respect to SARS-CoV-1. Mutations in SARS-CoV-2 Orf3a channel forming residues enhances the formation of a prominent the inter-subunit channel, which was not present in the SARS-CoV-1 Orf3a. This enhanced structural feature can be correlated with higher channelling activity in SARS-CoV-2 than in SARS-CoV-1. On the other hand, E protein is one of the most conserved protein among the SARS-CoV proteome. The water molecules form networks of electrostatic interactions with the polar residues in the E protein putative wetted condition (while no water channel formation is observed in the putative dewetted condition). This aqueous medium mediates the non-selective translocation of cations thus affecting the ionic homeostasis of the host cellular compartments. Ion channelling mechanism of SARS-CoV-2 viroporins potentially leads to ionic imbalance and $\mathrm{pH}$ change of subcellular compartments of the infected host cells causing membrane structured interfacial water and intracellularis function. Microsomal structures formed of the disrupted host membrane could be utilized in virion assembly and packaging. This ionic imbalance leads to increased inflammatory response in the host cell.

COVID-19 vaccine has been developed using various technologies by several Pharma giants like AstraZeneca, Moderna, Pfizer, Johnson \& Johnson along with several other lead candidates in line. The mRNA (Pfizer, Moderna) and the adenoviral vector-based vaccines (Astrazeneca, Johnson \& Johnson) use epitopes from the spike protein to generate an immunogenic response in the body and thus creating an immunogenic memory. But since spike protein is very much prone to mutations, these vaccines might lose their efficacies with the evolving viral genome. Hence, the other cellular events involved in the viral pathogenesis, which are more conserved phylogenetically, have become an important area of research. Ion channelling activity is one such feature which encompasses viroporins and their counter balancing host cellular responses which range from opposite directional ion flow to downstream disruptions of the host cell signalling pathways. 


\subsection{Spike Proteins}

Spike proteins (now on $S$-proteins) are trimeric class I transmembrane glycoproteins normally found as a homotrimer, and each monomer is composed of two subunits, named S1 and S2. The cleavage of the S protein into S1 and S2 is an essential step in viral entry into a host cell, and needs to occur before viral fusion with the host cell membrane. The $\mathrm{S} 1$ subunit contains a region, the receptor binding domain ( $\mathrm{RBD})$, that serves to bind to the target cell by adhering to the human angiotensin-converting enzyme 2 (ACE2), that serves as the virus receptor.

ACE2 is a mammalian transmembrane zinc-containing metalloenzyme [39], that cleaves angiotensin II (a vasoconstrictor peptide) into angiotensin (1-7) (a vasodilatator) (thus clearing angiotensin II and lowering blood pressure), mainly expressed in the lung, intestines, testis, arterial and venous endothelial (vascular system, including the molecular and humoral immune defence and lymphatic systems, acts as though it is a single organ), heart, kidney and alveolar epithelial type II cells (an active metabolic epithelial cells with a high density of mitochondria).

The structure of S-protein alone or in complex with ACE2 is being intensively studied. However, a detailed structural description of the potential interactions of the S-protein with lipids in the mammalian plasma membrane is currently missing [40]. This is a particularly relevant aspect since ACE2 is only the first target of interaction for the spike proteins and subsequent or parallel interactions with the surrounding membrane lipids are also pivotal for membrane fusion and viral entry in the host cell.

Compared to other coronaviruses, the S-proteins from SARS-CoV-2 have a very low dissociation constant $(14.7 \mathrm{nM})$ for the binding to ACE2, which makes SARS-CoV-2 highly infectious [40].

In [41] authors presented a strong argument that the S-protein by itself can cause a signalling response in the vasculature with potentially widespread consequences (which may lead to vascular wall thickening, mainly due to hypertrophy of the tunica media; enlarged smooth muscle cells may become rounded, with swollen nuclei and cytoplasmic vacuoles).

The binding of the S1 subunit to ACE2 via the RBD suppresses ACE2, causing a large increase in angiotensin-II (which can lead to pulmonary arterial hypertension), and is fundamental to convert the S2 subunit from a metastable pre-fusion state into a more stable post-fusion state, which triggers conformational changes that promote the fusion between the plasma membrane of the host cell and the virus envelope.

Spike's S1 subunit is able to significantly strip away lipids from the plasma host cell bilayer membrane, via a non-specific interaction (electrostatic manner, ion channelling activity, protein-protein interaction, electron-proton transductions and exchange are good candidates), causing a large change in the composition of the lipid bilayer, compatible with an increase of water content, disrupting 
and potentially entering directly through the host membrane, thereby allowing the viral RNA to be released into the host cell cytoplasm [40].

Endocytosis of Spike bound to ACE2 is one potential mechanism for viral entry, a mechanism that has been proposed for viral entry in some cell types [42].

Although the total structural charge of SARS-CoV-2 is positive, the structural proteins of SARS-Cov-2 are carrying varied total electric charge related to the amino acid content [27]. The E, $\mathrm{M}$ and $\mathrm{N}$ proteins are positive, while the surface S-protein is negative. The latter contains 99 positively, and 111 negatively, charged amino acids (AAs). The charged AAs in the S-protein of SARS-CoV-2 also exhibit some specific distribution. In the RBD region of the $\mathrm{S} 1$ unit the positive charge (cations) dominates in the protein interior (in general cations prefers protein interior) and the negative charge (anions) prevails on the surface exposed towards ACE2 receptor. The charged AAs in the S2 region preceding heptad repeats of the S-protein, exhibit a central concentration, and the imbalance of a positive charge. Both distributions of charged AAs may, in an electrostatic manner (eventually via compression of the membrane thickness due to electromechanical-deformation [43]), facilitate the coronavirus infection of the host cell.

The inhomogeneity of the electric charge distribution (dipole-like anionic-cationic charge inhomogeneity) shown when considering the interface of SARS-CoV-2 spike RBD and human ACE2, contribute to the occurrence of the resultant mesoscopic Coulomb forces between the S-protein and the ACE2 receptor. In the stricter border area there is an excess of negative charge with some isolated areas of positive charge, both in RBD and ACE2. The importance of the electric charge is somewhat confirmed by the evolution trend to conserve the total charge $q$ in the central region of RBD.

The long-range electrostatic Coulomb attraction may govern an initial approaching of the SARS-CoV-2 spike RBD and human ACE2, leaving electrostatic forces involved in the virus-cell approaching phase. Despite the spike and the receptor charge in the total are negative, the local multipolar (monopolar and dipolar) interactions may produce both attraction and repulsion of approaching proteins (at the RBD surface cationic peptides could be more binding than anionic ones, due to the prevalence of negative surface charge at the RBD). However, the final precise binding with the receptor also requires other types of physical interactions in the peptide-protein interface, mainly hydrogen-bonding with polar or amphiphatic amino acids along with biophysical and biochemical structured interfacial water stressors (see section two).

It is thought that the membrane permeation is dependent on the membrane fluidity, which in turn is dependent on the membrane lipid composition, cell microenvironment and the presence of charged phospholipid head groups [44]. Modulation of the membrane fluidity may arise due to the ease of movement of water molecules, and the dielectric constant of water, which is affected by endogenous and/or exogenous non-ionizing EMF [45]. 


\subsection{Early Stages of SARS-CoV-2 Infection in Broad Outline}

The entry pathway of SARS-CoV-2 into the human host and the engagement of target host cells can be summarized as follows (subsequent phases summarized in Figure 2):

- Virus enters the host 1) via upper respiratory tract (URT) inhalation of infected respiratory droplets or aerosol, 2) via contact with infected surfaces, such as skin-to-skin, and through touching an infected inanimate object then mediating it through the mouth, nose or eyes, and 3) via fecal-oral route.

- Virus "senses" the host environmental cues (a possible explanation is that virion ULF-EMF emission selectively tunes to ions resonance frequencies in cells and/or cell membrane in ways that affect the target cell's responsive ULF-EMF, the virion "detect" the emitted responsive signal and orientates towards the signal source) and move towards target cells (eventually by RNA

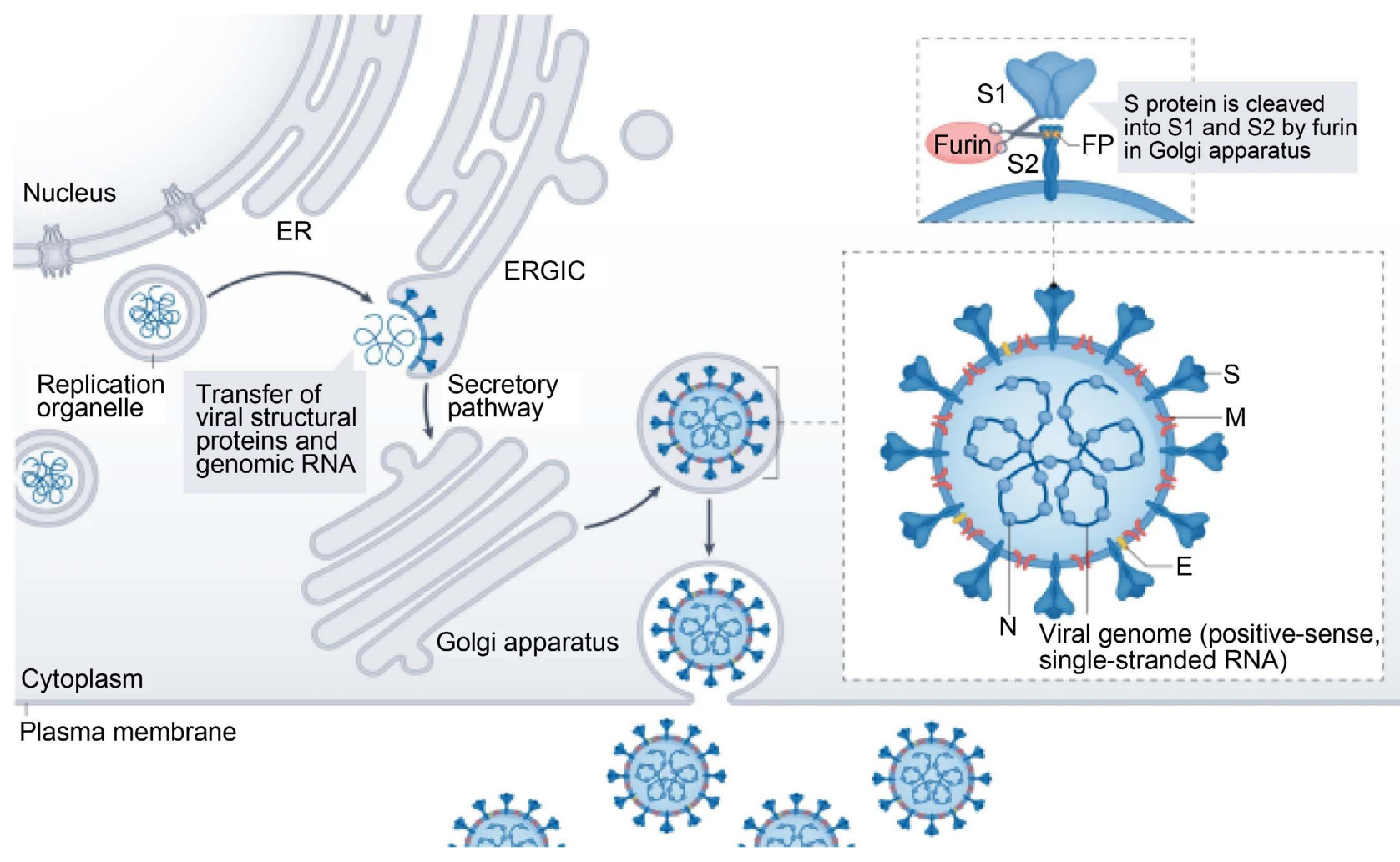

Figure 2. Mechanisms of SARS-CoV-2 entry into cells. Infection by a SARS-CoV-2 induces in the host cell perinuclear area the formation of new membranous structures of various sizes and shapes, which likely originate from the endoplasmic reticulum (ER) and as a whole are referred to as replication organelle. Viral structural proteins and genomic RNA synthesized at the replication site are then translocated through an unknown mechanism to the ER-Golgi intermediate compartment (ERGIC), where virus assembly and budding occur. While the N protein bound to the viral genomic RNA is packed inside the virion, the structural proteins $\mathrm{S}, \mathrm{E}$ and $\mathrm{M}$ are incorporated in the virion membrane. During biosynthesis and maturation in the infected cell, the $\mathrm{S}$ protein is cleaved by furin or furin-like proprotein convertase in the Golgi apparatus into the S1 and S2 subunits, which remain associated. In the new target cell, the S1 subunit binds the receptor and the S2 subunit anchors the S protein to the virion membrane and mediates membrane fusion. The $\mathrm{E}$ and $\mathrm{M}$ proteins contribute to virus assembly and budding through the interactions with other viral proteins. Assembled viruses bud into the ERGIC lumen and reach the plasma membrane via the secretory pathway, where they are released into the extracellular space after virus-containing vesicles fuse with the plasma membrane. FP, fusion peptide. Image source: Mechanisms of SARS-CoV-2 entry into cells https://www.nature.com/articles/s41580-021-00418-x. 
strand machinery resting on non-ionizing EM radiation interactions, namely electromagnetotaxis), resorting to retroactive, non-linear, biochemical and biophysical stimulus-response processes, which may refer to a less integrated but no less effective form of mixotaxis equipped with electromagnetotaxis. Airway and alveolar epithelial cells, vascular endothelial cells and alveolar macrophages are among its first targets of viral entry.

- Virus recognise (via electrochemical and ion-cyclotron resonance [14] interactions-see section three) the target cell ACE2 virus receptor.

- The S-protein is glycosylated by the host cellular glycosylation apparatus as it passes through the secretory pathway, before binding to the high-affinity receptor ACE2 [46] [47].

- The highly glycosylated Spike's S1 subunit RBD bound to the host cell ACE2.

- Direct interaction with the lipid bilayer on the cell surface occurs, lipids from the cell membrane are stripped away, causing a large change in the composition of the lipid bilayer, disrupting and potentially entering directly through the membrane [48].

\subsection{Host Cell Oxidative Stress Reactions}

Once the target host cell is engaged, SARS-CoV-2 cationic fusion peptides, (FP), a short peptide segment in the S-protein, which plays a central role in the initial penetration of the virus into the host cell membrane [49], ties up the heparan sulfate proteoglycans (HSPGs) on the host membrane's outer surface by charge neutralization [50], causing sulfation of the HSPGs [51] [52], exposing localized hydrophobic glycine-rich regions, and thus breaks up the HSPG-membrane complex [53] [54] that connects extracellular matrix components to the intracellular cytoskeleton. The resulting disconnection of the cytoskeleton from the plasma membrane has several adverse consequences, including impaired electrical conductivity of the cytoskeleton and microtubules and re-orientation of the cytoskeleton toward the cell nucleus, which may induce membrane fusion and accelerate viral mitosis. Once the host cell membrane's barrier is disrupted, penetration of the viral RNA into the host cell cytoplasm disrupts intracellular water structure, leading to unfolded protein response (UPR), unfolded DNA response, and excess reactive oxygen species (ROS) and reactive nitrogen species (RNS) production.

Viral pneumonia caused by SARS-CoV-2 induces overactivation of immune response in the lung tissues affected by virus replication. This pathological process is nearly always accompanied by disturbed redox balance and oxidative stress (OS). OS is typical for infection of human respiratory syncytial virus, rhinoviruses, and many other viruses. In a large number of pathologies, inflammation is known to be closely related to OS, one process being easily induced by the other [55].

After the virus enters the airways, its replication occurs, and the immune innate response begins with the activation of macrophage and dendritic cells via Toll-like receptors (single-pass membrane-spanning receptors usually expressed 
on sentinel cells such as macrophages and dendritic cells, that recognize structurally conserved molecules derived from invading pathogens) and Nucleotide-binding oligomerization domain (NOD) receptors (cytoplasmic pattern-recognition receptors for detecting invading pathogens) against the production of inflammatory cytokines and ROS/RNS.

Much of the cytosolic water is created as a byproduct of mitochondrial respiration and electron chain transport. This metabolism makes heat (biophotons emission and phonons transfer) and the density of electrons flowing along the inner mitochondrial membrane correspond the electronegative charge (which makes proteins hydrophilic). Mitochondria transport protons into the intermembrane space, and their diffusion into cytosol leads to generation of a strong static electric field and water ordering.

When mitochondrial function slows, the infrared light and electronegative outputs of the cell drop and so does the volume of structured interfacial water (CD/EZ) [56].

In presence of dysfunctional mitochondria, the surrounding structured interfacial water's layer reorganize with a reversed orientation of the electric field, which enables transport of electrons released into cytosol. As biological water occupies $70 \%$ of the cell volume, it is capable of releasing a huge amount of electrons into the cytosol. Free electrons increase conductivity which causes damping of cellular electromagnetic field (EMF). The mechanism of damping electromagnetic oscillations generated by microtubules [57] may explain the disturbed organization in cells with dysfunctional mitochondria [58] [59].

When $\mathrm{O}_{2}$ is absent during periods of irregular cell hypoxia in mitochondrial energy synthesis, the generation of excess electrons can develop free radicals (mitochondria are major sources of free radicals) or excess protons can produce acid, the latter case being enhanced if ROS hydrogen peroxide $\left(\mathrm{H}_{2} \mathrm{O}_{2}\right)$ produce ROS hydroxyl radical $\left(\mathrm{OH}^{*}\right)$ when exposed to transition metal cations such as divalent ferrous iron or $\mathrm{Fe}^{2+}$ common to the heme molecule ${ }^{3}$. Free radicals formed by limited $\mathrm{O}_{2}$ can damage lipids and proteins and greatly increase molecular sizes in growing vicious cycles to reduce oxygen availability even more for mitochondria during energy synthesis. Further, at adequate free-radical concentrations a reactive crosslinking unsaturated aldehyde lipid breakdown product can significantly support free-radical polymerization of lipid oils into rubbery gel-like solids and eventually even produce a crystalline lipid peroxidation with the double bond of $\mathrm{O}_{2}[60]$.

The consequent spread of inflammatory cytokines and ROS/RNS to the blood has two consequences: 1) erythrocytes are damaged by ROS and other inflammatory mechanisms leading to denaturation of hemoglobin and iron metabolism dysregulation (ferroptosis) with release of toxic free iron ion [61]; and 2) ac-

${ }^{3}$ Heme proteins are important biological molecules that catalyze radical reactions, and thus they can induce proton spin coupling dependent local field effects on the involved intermediate free radical substrates. Heme proteins are the antioxidant enzymes catalases and peroxidases, the oxygen transporters hemoglobin and myoglobin, and all mitochondrial respiratory chain (and photosynthetic electron chain) cytochromes. 
tivated macrophages and neutrophils produce respiratory bursts generating superoxide radicals and $\mathrm{H}_{2} \mathrm{O}_{2}$ leading to OS. OS plus free iron ions converts soluble plasma fibrinogen into abnormal fibrin clots in the form of dense matted deposits (DMD, resistant to the enzymatic degradation; blood clots), leading to disseminated intravascular coagulation (microthrombosis in the vascular system and the pulmonary microcirculation), cell hypoxia and endothelial dysfunction (both microvascular and endothelial dysfunction share a common initial pathophysiology).

The cytokines storm occurs through damped or impairment chemokine regulation. The severity of COVID-19 can be stratified based on plasma/serum chemokine levels. Chemokines are a large family of small secreted cytokines that coordinate leukocyte trafficking and activation, thereby regulating diverse physiological processes, including development, inflammation, immune responses and wound healing.

After this scenario is established, the cytokines storm induces OS via macrophage and neutrophil respiratory burst activity, and OS induces the cytokines storm. This cycle provokes serious tissue damage independent of the virus. Low hemoglobin-carrier, high lung proteinaceous exudate leads to pulmonary hypoxia, cytopathic hypoxia and endothelium damage, and disseminated coagulation results in multiple organ collapse.

Several signs and changes in clinical and laboratory examinations presented by patients with severe COVID-19 have been described. The changes observed include decreased total lymphocytes, prolonged prothrombin time, elevated lactate dehydrogenase, cellular immune deficiency, coagulation activation, myocardial, hepatic, and renal injury and endotheliitis in the lung, heart and small bowel.

Although the mechanisms involved in these changes are still under research, OS by ROS is related to all the main changes observed and could be the connecting point that unites all these events [62].

Overall, a model has emerged in which SARS-CoV primarily infects lung epithelial cells to undergo replication, followed by infection in macrophages, with induction of chemokine expression in both cell types. Next, chemokines mediate recruitment of additional macrophages, neutrophils and $\mathrm{T}$ cells. Upon activation, these leukocytes contribute to an exuberant immune response which may involve further production of chemokines, potentially contributing to immunopathological damage in the lung and development of acute respiratory distress syndrome (ARDS) [63].

\section{Discussion}

Oxidative stress is defined as an imbalance between toxic ROS and antioxidants in favor of oxidants, leading to a disruption of redox signaling and/or irreversible oxidative damage to lipids, deoxyribonucleic acid (DNA) or proteins. Oxidative damages are involved in the development of different pathologies including cancer, cardiovascular, neurodegenerative and lung diseases.

Most organisms rely on the role of oxygen as a terminal electron acceptor for 
efficient energy production in the form of ATP. Increased intracellular levels of oxygen, however, are potentially toxic. This toxicity is mainly due to partially reduced forms of $\mathrm{O}_{2}$, since the $\mathrm{O}_{2}$ molecule per se has low reactivity. The molecules and radicals formed by the incomplete reduction of oxygen are termed ROS and RNS. ROS/RNS commonly formed in vivo include the superoxide radical anion $\left(\mathrm{O}_{2}^{--}\right)$, hydrogen peroxide $\left(\mathrm{H}_{2} \mathrm{O}_{2}\right)$, and the hydroxyl radical $\left(\mathrm{OH}^{\circ}\right)$ [64] [26].

ROS/RNS free radicals play important roles in regulation of cell survival. In general, moderate levels of ROS/RNS may function as signals to promote cell proliferation and survival, whereas severe increase of ROS/RNS can induce cell impairment and death. Under physiologic conditions, the balance between generation and elimination of ROS/RNS (which is known to rest on the free radical pair mechanism [63]) maintains the proper function of redox-sensitive signaling proteins. Normally, the redox balance ensures that the cells respond properly to endogenous and exogenous stimuli. However, when the redox balance is disturbed, OS can result in damage to proteins, lipids, nucleic acids, and contribute to disease development.

\subsection{Structured Interfacial Water: Coherence Domaine and Exclusion Zone}

In biological systems, liquid water interacts not only with small solutes but also with many larger, extended hydrophilic and hydrophobic surfaces, such as those of proteins, nucleic acids, various organelles, and cell membranes [65]. Results of inelastic incoherent neutron scattering studies of several cell and tissue types suggest that ca. $20 \%-30 \%$ of the total (intracellular plus extracellular) water in these systems is interfacial water, i.e. water located within $1-4 \mathrm{~nm}$ of these surfaces (thus, biological water can be considered as interfacial water because there is almost no point in an organism that is not far more than a fraction of a micron from a surface), with bulk water comprising the remaining 70\% - 80\% [66] [67].

Water at an interface, as with the atmosphere, has a surface tension due to the polar interactions of water with other water molecules at the interface surface. This clustering imparts a crystalline like property to the water. In the bodies of living organisms, the clusters form hydration layers around biological molecules. It is known from electronics that different patterns which contain in-formation ${ }^{4}$ result within a cluster depending upon its structure. Thus, depending on its structure, each molecule has an oscillatory pattern (resonance frequency) that can be determined by spectroscopy. It is known, through spectrographic analysis, that water and other dipole molecules are able to be entrained to exogenous ${ }^{4}$ It must be stressed out that the physical concept of "information" it has absolutely nothing to do with that of "data transmission", and even less with that of "transmission of messages containing a (semantic) meaning". The former (physical concept) consider "information" as a measure of coherence or structural "complexity" of surrounding system related to various entropic processes in physical world, that is the measure of in-formation amount, related to a certain object, may be a complexity of its internal structure (negentropy), while the latter (IT concept) consider "amount of information" as frequency characteristic of code letters-signals, that is improving of messages coding and decoding methods and solving of other questions related to optimization of technical communication systems operation. 
oscillatory patterns by rearranging their cluster patterns. The cluster rearrangements then resonate with the entraining frequency.

Interfacial water assumes a glassy appearance, i.e. semi-crystalline, and has been studied by several researchers [68], suggesting the possibility that it is of a different phase (Pollack's four phase [69]) from that of common water in the liquid state [70] [71].

All biological envelopes, from cell membrane to epithelial tissue, contain this aqueous phase in a liquid-crystalline state or are perfused by it. It is water in a particular phase of quantum organization that close to charged hydrophilic surfaces is confined in Coherence Domains (CDs) of about $100 \mathrm{~nm}$ in diameter at ambient conditions [72]) and in layers of Exclusion Zone [73] [74] (which may be regarded as long-range ensembles of $\mathrm{CDs}$ ), and gives it a high capacity to:

- retain electronic charges, in the form of vortical excitations of quasi-free electrons, storable as energy reserve;

- induce an electronic and protonic long-range and long life excitation of the different molecular species available, enabling their selective activation and mutual attraction;

- convert mechanical vibrations (phonons) in quanta of electromagnetic energy (photons) and vice versa (piezoelectric effect).

EZ interfacial water shows specific properties which may be summarized as follows [73] [75]:

- EZ interfacial water is considerably more viscous than bulk water (about 10-fold) [76].

- EZ interfacial water has a negative electric potential (up to $200 \mathrm{mV}$ ) with respect to the neighboring normal bulk water; thus the pair EZ interfacial water-bulk water is a redox pile. This property of EZ interfacial water could account for the source of electron excitations.

- Protons concentrate at the boundary between EZ interfacial water and bulk water.

- EZ interfacial water exhibits a peak of light absorption at $270 \mathrm{~nm}$; it emits fluorescence when excited by light having this wavelength.

- The illumination of EZ interfacial water by light (especially IR radiation) increases the depth of the layer.

- EZ interfacial water cannot host solutes.

The above list of properties appears quite mysterious in the frame of conventional ideas about liquid water (monophasic liquid), while is well explained in the frame of QED (biphasic liquid) ${ }^{5}$.

\footnotetext{
${ }^{5}$ Neither classical nor standard quantum theory predicts quantum coherence for water, largely because they ignore quantum fluctuations and the interaction between matter and the vacuum electromagnetic field (VEMF), which are taken into account in Quantum Field Theory (QFT). The conventional $a b$ initio approaches to water, based just on Quantum Mechanics (QM), describe it as a monophasic liquid. On the contrary, the conceptual frame of QFT admits infinitely many ground states (vacua), each one corresponding to a particular function describing the expectation value of the involved field. Quantum coherence for water appears quite mysterious in the frame of conventional ideas about liquid water (monophasic liquid), while is well explained in the frame of quantum electrodynamic QED (biphasic liquid) [77].
} 
The Coherence Domaine is a resonating cavity produced by a self-trapped EMF (a self-produced cavity resonator for the EMF), whose size is just the wavelength $\lambda$ of the trapped EMF, that ends up trapping the field because the photon acquires an imaginary mass, so the frequency of the CD EMF becomes much smaller than the frequency of the free field with the same wavelength.

Within the CD water molecules oscillate between the ground state and an excited state close to the ionizing potential of water and, therefore, contain close to a million almost-free electrons. That means $\mathrm{CD}$ is most likely negatively charged at the periphery close to or at the surface of its domain (at the same time, positively-charged protons are present just outside the $\mathrm{CD}$ ).

According to [65] structured interfacial water, namely biological water, promotes life-enabling biological processes by:

- promoting electrical conductivity at biological interfaces, thereby facilitating metabolism and voltage differences maintained by intracellular organelles;

- absorbing, storing, and emitting electromagnetic energy, enabling storage and transmission of energy and in-formation;

- overcoming the $\mathrm{kT}$ or "thermal diffusion" problem and solving the intracellular crowding and molecular self-assembly problems by way of chirality (handedness of molecules) and magnetization.

Therefore, pure bulk liquid water consists of two interspersed phases, coherent and incoherent, having widely different dielectric constants (that of the coherent phase is 160 , due to the high polarizability of the coherently aligned water molecules that are oscillating in concert; while the dielectric constant of the incoherent state is about 15) and appears to behave as an active medium able to perform through ultra low frequency-electromagnetic fields (ULF-EMF).

In the coherent phase, the water molecules oscillate between two electronic configurations (that of the $\mathrm{CD}$ and that of its matrice) in phase with a resonating $E M F$. The EMF that are trapped within the $\mathrm{CD}$ of water and within its coherent matrice [77], produce electromagnetic potentials (EP) that regulate the phase $\Phi$ of the entire system, which in turn gives rise to selective attractions between the molecules of the solute.

Contrary to the objects described by Classical Physics, a coherent quantum system is not defined in isolation, but gets defined by the array of its relationships. The phase $\Phi$ is connected with the EM potential in a mutual relationship so that we could be able to change the phase $\Phi$ of a biological organism by applying an EM potential [78] [79] [80].

We could interpret the biological effectiveness of very- and ultra-weak EM and magnetic fields just by assuming that the agent at work in the interaction is not the field but the potential and the mechanism of interaction is the phase-sharing ${ }^{6}$.

${ }^{6}$ Exogenous and endogenous electromagnetic signals can be selectively damped by tissues and cells, according to their being or not in phase with the possible oscillatory motion of the system's components. This specific phase-matching feature operates as a very selective mechanism, a sort of filter discriminating among perturbations and stimuli acting on the system, thus protecting it against any noisy perturbative background or even strong actions, which, however, are out of phase with the oscillatory motions allowed by the system's inner dynamics [81]. 
That is, biological organisms, being coherent, can interact with environment in two basically different ways:

- through the conventional exchanges of energy which amount to the application of mutual forces. This mechanism of interaction obeys the causality principle, since energy cannot travel faster than light (c);

- through the sharing of the phase $\Phi$ with other coherence based systems (biological organisms) and with the environment itself, which leads to relationship systems based on resonance (phase-matching means oscillatory resonance). The phase $\Phi$ velocity is not bounded above and can be larger than light speed $c$.

\subsection{Coherent Water and Biological Structures Are Semiconductors}

Because coherent water is excited water with a plasma of almost free electrons, it can easily transfer electrons to molecules on its surface. Non-coherent water is an almost perfect insulator and chemically an oxidant, coherent water is a semiconductor and chemically a reducer; the interface coherent water/non-coherent water is therefore a redox pile [74] and a difference of electric potential can be found across it (estimated to be included in the interval between 50 and $100 \mathrm{mV}$ ) [82].

Various biological structures, e.g. keratin, collagen, alpha and beta sheaths of proteins, genes and so forth are semiconductors and possess the ability to activate charges without participation of ions [83].

They have been conceived to be piezoelectric (the ability to generate an electric charge in response to applied mechanical stress) and pyroelectric (the ability to generate an electrical potential in response to applied thermal stress), which means they possess the capacity for thermal and mechanical polarization.

Whereas, these structures may form an uninterrupted reticulum that may act as quasi-crystalline piezoelectric-pyroelectric networks, capable of converting electromagnetic oscillations to mechanical or thermal stress, or vice-versa. Hence, basic organic compounds and the whole organism are subjected to electrostriction, i.e. the mechanical deformation of a dielectric (insulator) in the presence of an electric field, and become quantum generators of phonons or acoustic waves (a phonon being a quantized mode of vibration). The transition from the induced state to the basic state has got thus two possibilities: the generation of photon or the generation of phonon. Thus, photon-phonon transductions arguably must exist in all material bodies, especially because all ordinary matter is nothing else than condensed electromagnetic field.

Interfacial water EZ layer is an electron-donor, namely a reducer, whereas normal water is a mild oxidant, consequently the interface EZ-water/bulk water is a redox pile, where the redox potential could have a jump of a fraction of a Volt. Hence, life could be defined as the dynamics occurring between two levels of the electron clouds of water molecules: an excited state and a ground state. It is just this electrondynamics at the origin of the singular redox properties found 
in the water in living matter [84].

In this conceptual frame life can be linked to a little electric current going round and round. It is apparent that here electromagnetic fields find a place within the biological dynamics. Electromagnetic fields are just able to couple with the current of electron excitation producing important consequences on the biochemistry which is just governed by this electron excitation.

At micro- and mesoscopic level life is a result of all the chemical, electrical, magnetic, optical and acoustic events occurring in the living organism, in the system of organic-like semiconductors, piezo- and pyroelectrics. Therefore, life takes place not in a chemical or electronic system, but to some extent among these two processes, where photon-phonon and electron-proton transductions and exchange play a central role.

\subsection{The Self-Driven Liquid-Flow System}

Proton currents and concentration gradients are ubiquitous in all biological systems and play essential roles in a number of physiological processes [85]. The most striking example is oxidative phosphorylation in mitochondria in which proton gradients serve as a means to translate the energy from oxidation of glucose during the Kreb's cycle into ATP, the biological energy currency [86] [87].

Thanks to the hydrogen-bonded chain mechanism [88] [89], called the Grotthuss mechanism, protons tunnel from one water molecule to the next via hydrogen-bonding [90].

Surprisingly, excess protons can create their own pathways, water-wires, before protons can migrate along [91].

Water electricity is special in that it also involves the movement of positive charges associated with protons. According to quantum electrodynamic (QED) not only do electrons of the hydrogen-bonds fail to conform to the classical electrostatic model, the protons also are quantum mechanical. Changes in the redox potential (oxidation state or electronic configuration) or proton transfer equilibria of a chemical species can influence each other, thereby these two chemical reactions often occur in association. This fundamental concept of chemistry is best exemplified by the Nernst Equation which relates the variation of the aqueous oxidation/reduction potential of chemical reactions with the $\mathrm{pH}$ when protons are involved.

It is known that cells can exist and perform particular functions in complex environments within a particular range of temperature and $\mathrm{pH}$ conditions [92].

The $\mathrm{pH}$ level is different for different parts of the body. Thus, for example, in order to decompose food to basic components, the stomach maintains an acidic environment. The cells, covering inner walls of stomach, must be resistant to these extreme conditions and the proteins and receptor-ligand complexes within should be able to perform their functions. Another example is immunocompetent cells, such as monocytes and neutrophils. While being activated, they produce reactive oxygen species (ROS) that acidify the environment. Thus, the 
change in $\mathrm{pH}$ is used as a powerful weapon against pathogen organisms (an acidified environment which instead favors SARS-CoV-2 pathogenicity). In addition, these cells perform phagocytosis, at which monocytes and neutrophils capture the pathogens inside phagosomes inside cells. Furthermore, the $\mathrm{pH}$ value is not constant throughout the cell, compartments of the cells can have widely differing $\mathrm{pH}$.

In a more general sense, the thermodynamic correlation between electron and proton transfer establishes that the redox potential of a chemical species can affect its acid-base equilibria, that is, its $\mathrm{pK}_{\mathrm{a}}$ (protonation state) [93]. As pointed out earlier, SARS-CoV-2 main protease $3 \mathrm{CL} \mathrm{M}^{\text {pro }}$ is indispensable for virus replication and do affect the host cell protonation state by increasing its acidity degree.

This concept constitutes the thermodynamic basis of proton-coupled electron transfer (PCET) reactions [94] [95], also called Concerted proton electron transfer (CPET), in which proton and electron movement are intercorrelated and were proton transfer?

- Is involved in regulating enzyme reactions within the cell, where metabolic reactions are predominantly of a redox nature.

- Proton currents may well flow throughout the extracellular matrix, and linked into the interior of every single cell through proton channels.

- Proton currents could flow from the most local level within the cell to the most global level of the entire organism.

- Protons (reducing power) give a boost of energy where it is needed.

- Protons can flow directly along the membrane within the interfacial water layer, from proton pump to ATP synthase, both of which are embedded in the membranes.

Proton currents and concentration gradients play essential role also for it can mimic a motive-like pump that turns any biological liquid flow collagen-related system in a self-driven liquid-flow system (S-DLF-S). That is a light-driven-flow [96].

Collagen is the most abundant protein in the organism, and is known to form liquid crystalline mesophases (liquid crystals are states or phases of matter in between solid crystals and liquids, hence the term, mesophases). It is the main protein in the extracellular matrix and connective tissues (connective-collagen membrane system happens to be composed almost entirely of hydrophilic material, which are defined by the density of electrons on their surfaces, and the protons formed when (a) ultraviolet to infrared light energy, (b) water, and (c) hollow hydrophilic surfaces come together in space and time and may thus account for the liquid crystallinity of living organisms as a whole, facilitating short and long range collective coherent correlation throughout the body [70]).

\footnotetext{
${ }^{7}$ Electron transfer (ET) and proton transfer (PT) denote the electron/proton flow process with an electrolyte, which is ionically conducting and which can function as an electron/proton sink or source via a redox process. The electrolyte also provides charges, which screen the change in electrical potential due to the electron/proton flow and makes a charge balance possible.
} 
Cells and complex molecules suspended in the blood avoid agglomeration through a negative charge field maintained in the immediate surrounding space. The rate at which a charged particle suspended in a medium will travel in an applied electric field is an important measure of colloidal stability in the medium, and is associated in physics with the electrical potential drop from the particle surface across the bound fluid, to the interface where the liquid begins to flow under the shear stress, namely Zeta Potential (ZP), a measure of the net charge density of a particle (also a measure of surface charges of virus particles which characterize the viral electrostatic properties), i.e. the potential at the surface boundary between the stationary fluid and the liquid that is moving with the particle. A high negative value for ZP (if a nanoparticle in a dispersed system moves with electrophoresis to a positive electrode, its charge is negative and vice versa) is essential for maintaining blood as a colloidal suspension.

Blood vessels are made of hydrophilic proteins, which structure the water in blood and form the EZ gel-like state observed at nanoscale in the interaction between water and hydrophilic surface. The negative ions (also in the form of vortical excitations of quasi-free electrons) propel the protons through the middle and drive perfusion. Once the blood plasma is electrically structured, the negative electrical value for ZPof red blood vessels builds and prevents them from clustering ${ }^{8}$. A systemic lowering of serum ZP mediated by exogenous cationic surfactant administration, together with lowered bioavailability of certain endogenous sterol sulfates, sulfated glycolipids, and sulfated glycosaminoglycans, which are essential in maintaining biological equipose, energy metabolism, membrane function, and thermodynamic stability in living organisms, leads to subsequent inflammation, serum sickness, thrombohemorrhagic phenomena, colloidal instability, and ultimately even death.

According to [98] [99], the highly ordered collagen water chains (linked to Pollack's fourth phase of water), are reminiscent of those seen in carbon nanotubes $(<5 \mathrm{~nm}$ diameter).

Under certain conditions, Pollack's team have been able to visualize EZ water inside tunneled gels and hydrophilic tubes made of Nafion immersed in an aqueous microsphere suspension. Videos show a steady flow of microspheres and water along that interior channel, the so-called "self-driven" flow (see also [100]).

Light drives that flow; increasing light speeds it by up to five times. Hence, the light driven-flow phenomenon is general; and, it is driven by light. They found that the flow resulted from the protons generated as consequence of $\mathrm{EZ}$ growth. Those protons lie in the tunnel's central core. Repulsion among protons creates a pressure, which pushes the protons out of one end of the tube or the other.

\footnotetext{
${ }^{8}$ In an ideal system like blood, we want all particulates to have a like electrical charge. If the particles have no electrical charge, the various particles will clump together and form sludge. Therefore, the higher the Zeta Potential, or negative electrical charge, the better the dispersion of particles in suspension. The high Zeta Potential on particles entering the bloodstream may help to increase the dispersion or discreteness of blood cells by helping to enhance the electrical charge on blood colloids which include blood cells [97].
} 
Once that flow begins, additional water gets drawn in from the opposite end of the tube, perpetuating the process.

Pollack's hydroelectric EZ reveal, at least in vitro [96], that IR/UV energy from light outside the organism, and possibly also from biophotons derived from internal exothermic metabolic reactions, may transform simple hydrophilic tubes and vessels, including arteries, veins and capillaries, lymphatic ducts, renal tubules, sweat glands, ureters, tear ducts, Eustachian tubes, respiratory alveolar ducts, bile ducts, cerebrospinal fluid-flow system, axons, etc., into the equivalent of mechanical pumps but without a motor, i.e. into S-DLF-S. That is, all of these tubes and vessels, as well as any other collagen-related liquid-flow system, need pumps to actively move the associated fluids within.

The pumping action exerted, e.g. by the cardiac activity on blood and on CSF-FS circulation, as well as the pumping action exerted by the contractile activity of the striated and smooth muscles, behave as pumps and actively move the body fluids, but their efficiency would be drastically reduced without the active contribution of the S-DLF-S (Figure 3) engaged by proton currents and concentration gradients. S-DLF-Sengaged in blood circulation within the blood vessels is an example: red blood cells flow in the core of vessels and are excluded from the periphery. This feature is long known, and is generally attributed to hydrodynamic effects; but the evidence above implies that it might arise instead from the EZ proton currents and concentration gradients [99].

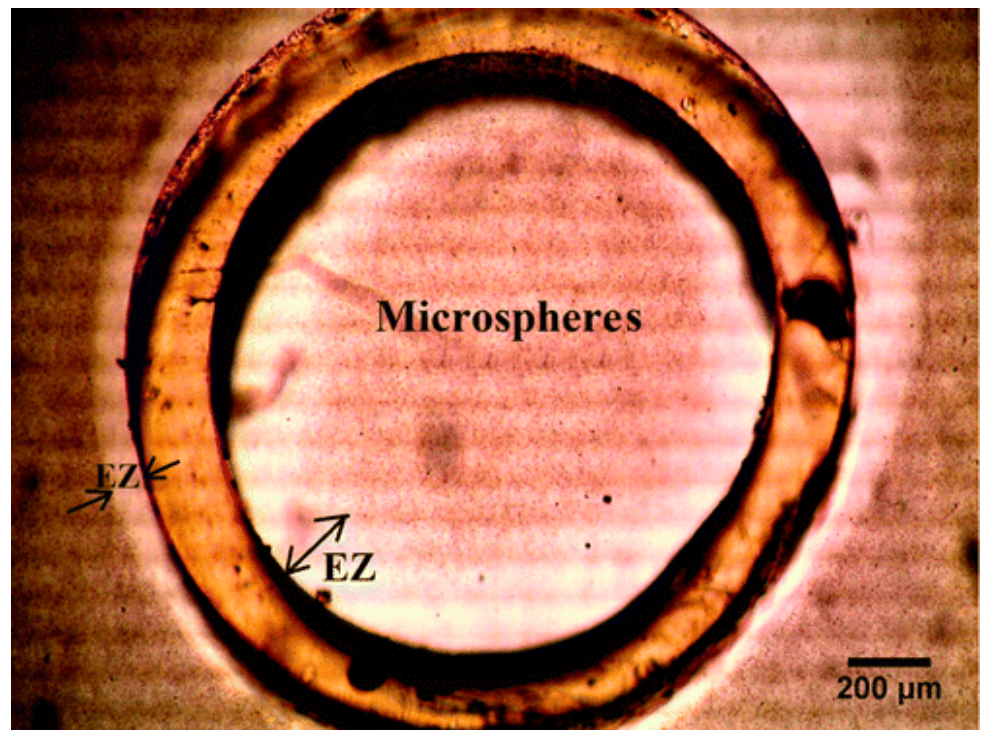

Figure 3. Self-driven flow was observed in Nafion and other hydrophilic tubes immersed in water. The intratubular flow was generated when water came in contact with the tube's hydrophilic surfaces. Flow characteristics were studied in tubes of varying size, exposed to light of different intensities and wavelengths. The results lead to the hypothesis that the flow is driven by a high concentration of protons accumulating inside the tube, creating an axial proton gradient between the inside and outside of the tube. It is also demonstrated a faster flow under incident light, particularly at UV wavelengths, implying that proton generation may be driven by light. Credit [96]. Image source: https://pubs.acs.org/doi/abs/10.1021/la4001945?journalCode=langd5. 
Thus, if we exclude the mechanical action exerted by the smooth and striated muscles, the main motor force of any biological liquid-flow system is generated across biological membranes and connective-collagen membrane system, that is under EZ/CD electrodynamics laws, by proton currents and concentration gradients.

\subsection{Viral ULF-EMF as Host Cell Stressor}

Electromagnetic signals are endogenously generated at different levels of the biological organization and, likely, play an active role in synchronizing internal cell function or local/systemic adaptive response [101]. Consequently, each adaptive response can be described by its specific electromagnetic pattern and, therefore, correlates with a unique and specific electromagnetic signature.

According to QED, biological water can be viewed as an unstable balance between coherent and incoherent components, that is structured and bulk water. The molecules of water, the ions and the biomolecules of the coherent component form extended mesoscopic regions, called Coherence Domains (CDs) and Exlusion Zones (EZs), where they synchronously oscillate with the same phase, i.e. they oscillate in unison between two selected levels of their spectra in tune with a self-produced coherent EMF having a well defined frequency, dynamically trapped within the CD/EZ. Extended coherent mesoscopic regions are surrounded by the incoherent component where molecules oscillate with casual phases relative to one another.

It is possible to induce by an externally applied field (either hydrodynamical or electromagnetic) or also by a chemical stimulation, coherent excitations of $\mathrm{CD} / \mathrm{EZ}$ that give rise to electric currents circulating without friction within the CD/EZ: as a consequence magnetic fields are produced. A resonating magnetic field thus is able to extract the ions from the orbit and push them in the flowing current [102].

Accordingly, if an exogenous source of non-ionizing very weak low frequency electro-magnetic field, e.g. that of a virus or bacteria [103], enter an organism and interact with target cells by matching the ion cyclotron frequency resonance of a particular charged molecule, an intrinsic weak magnetic field is generated by ion currents in the cell. One of the main hypotheses currently available to explain this interaction is the parametric resonance Lednev's original model effect.

The Ion Parametric Resonance (IPR) theory, first proposed by Lednev (1991) and then by Blanchard and Blackman (1994) [104], is one of the most discussed models of low frequency electromagnetic field (LF-EMF) interaction with biological systems. The assumptions of this theory are based on the Ion Cyclotron Resonance theory (ICR), first described by the American physicist Abraham Liboff (1984), speculating that the physiological activity of certain important ions can be altered when the frequency of applied time-varying magnetic field is equal to the frequency of ion motion in a static magnetic field. Ions in IPR model are represented by a harmonic oscillator, bound to a specific location at the 
surface of the cell membrane, and application of combined magnetic field should alter its oscillations.

SARS-CoV-2, as any other virus, does emit and absorb ULF-EMF [17]. Given the extremely reduced nature of viral proteomes, ULF-EMF activity can rely on hydrogen-bonds (HBs), as piezoelectric centers of energy transduction and EM radiation emission centers, and on the single- or double-stranded nucleic acid machinery [105] [106], surrounded by a protein coat, which is everything a virus is made of.

It can be assumed that in its approach to the target host cell SARS-CoV-2 emits a stressful ULF-EMF [104] that may influence ionic fluxes throughout cellular membrane, before electrostatic interactions occur [107], activating the cellular phase-matching feature (see footnote 6), generating (also via plasma-membrane-localized redox signalling) an alert response in the cell membrane interfacial water phase (CD/EZ) which activate redox-responsive intracellular signaling and anti-inflammatory scavenging systems.

However, membrane ionic fluxes may be influenced by ULF-EMF in two non-mutually canceling ways, one based on QED and the other on QM.

Circular orbits of ions dissolved in water are localized on the surface of CD/EZ. The frequency of ionic orbits, called cyclotron orbits [108] [109], are proportional to a static EMF resulting from the vector addition of all EMFs present in the environment [110].

If an oscillating EMF with a frequency similar to the cyclotron overlaps the static EMF, ionic orbits are altered and ions change their position as to the surface of the interfacial water coherence domains. If domains are not next to the cell membrane, ions stop in the non-coherent water region and they are replaced by other ions. If domains are next to the cell membrane, ions go across it, thanks to the potential difference between the two side of the cell membrane. That is. Despite many physiologic oscillating EMFs exist into the human body providing the preservation of the structured interfacial water, if an exogenous EMF with a frequency matching the cyclotron frequency of a ionic species is present in the environment, that ionic species will be influenced by it and non-physiologic ionic fluxes through the cell membrane can occur causing membrane imbalance [111] [112] [113].

Another possible mechanism, based on Quantum Mechanics, which can be intertwined with the previous one, is the dissociation of ion-protein complexes due to weak oscillating EMFs in the presence of a static electromagnetic field. In this case there is an imbalance between intra and extra-cellular ionic concentration which originates metabolic disorders and high stress levels [114].

Therefore, long-range ULF-EMF interference events would precede the earliest steps in infection and infectivity by activating redox-responsive intracellular signaling and anti-inflammatory scavenging systems. Viral ULF-EMF would act as host cell stressor [103] [115], predisposing the target cells to infection and facilitating infectivity [116], by affecting membranal protein distribution, pro- 
moting ROS/RNS production, causing exogenous interfacial water stress (EIWS) [65] [117], disrupting structured interfacial water phase (CD/EZ) in favour of bulk water, stabilizing free radicals in such a way as to permit their dispersion rather than their return to the ground state, leading to localized water hydrophobicity, unwetting, increased water tension and membrane "softening".

\section{Conclusions}

Viruses act as parasites; they infect and either replicate within the host cell or integrate within the host genome. There are many direct and indirect causes of viral pathogenesis, but given the sheer numbers of viruses within a holobiont (the functional association between a host, prokaryotic, eukaryotic, and viral entities within a particular environment is the holobiont), and the limited pathogenesis that actually occurs, it seems more likely that viral pathogenesis is not as common as viral commensalism and mutualism [118]. A virus can be commensal, the virus benefits while host fitness is unaffected. A virus can be mutualistic, in which both organisms benefit and fitness increases. Such viral associations may provide advantages that promote evolution and biodiversity. One virulent virus among a sea of non-virulent viruses does not equate to pathogenesis. Unless transmission and recovery rates are high, pathogenicity may be an evolutionarily poor strategy for viral survival. More likely, pathogenesis is the exception and not the rule, with more instances being discovered of viruses having cooperative roles with the host [119].

SARS-CoV-2 belongs to viruses whose lytic/virulent lifestyle involves the infection, replication, and lysis of the cell, leading to the death of the cell and release of viral progeny. The earliest phase of its lytic/virulent lifestyle involves sensing and moving towards target cells, likely resorting to retroactive, non-linear, biochemical and biophysical stimulus-response processes (mixotaxis) equipped with electromagnetotaxis. In its approach to the target cell, specific ion effects may be triggered by electrostatic and electrodynamic forces. The latter likely consist of the emission of a long-range ULF-EMF which influences target ionic fluxes throughout cellular membrane generating an alert response within the cell, before electrostatic interactions occur. Therefore, it is suggested that the earliest virus-host interaction would rest on long-range electromagnetic events influencing the redox potential of the target host-cell's membrane chemical species, that is, affecting its acid-base equilibria, hence inducing a cellular stress response (plasma-membrane-localized redox signalling) with reactive oxygen species (ROS) and reactive nitrogen species (RNS) production. Accordingly, redox-responsive intracellular signaling and anti-inflammatory scavenging systems would be remotely (pre) activated by non-ionizing interference phenomena, before virus-cell come together, followed by electrostatic and chemical events that provoke a branching, cascade-like, chain of reactions.

\section{Conflicts of Interest}

The author declares that this work was conducted in the absence of any com- 
mercial or financial relationships that could be construed as a potential conflict of interest.

\section{References}

[1] SenGupta, S., Parent, C.A. and Bear, J.E. (2021) The Principles of Directed Cell Migration. Nature Reviews. Molecular Cell Biology, 22, 529-547. https://doi.org/10.1038/s41580-021-00366-6

[2] Barriga, E.H. and Theveneau, E. (2020) In Vivo Neural Crest Cell Migration Is Controlled by "Mixotaxis". Frontiers in Physiology, 11, Article No. 586432. https://doi.org/10.3389/fphys.2020.586432 https://www.frontiersin.org/articles/10.3389/fphys.2020.586432/full\#B62

[3] Binhi, V.N. and Prato, F.S. (2017) Biological Effects of the Hypomagnetic Field: An Analytical Review of Experiments and Theories. PLOS ONE, 12, Article ID: e0179340. https://doi.org/10.1371/journal.pone.0179340 https://www.ncbi.nlm.nih.gov/pmc/articles/PMC5487043/

[4] Funk, R.H.W., Monsees, T. and Özkucur, N. (2009) Electromagnetic Effects-From Cell Biology to Medicine. Progress in Histochemistry and Cytochemistry, 43, 177-264. https://doi.org/10.1016/j.proghi.2008.07.001

[5] Zhadin, M.N. (2001) Review of Russian Literature on Biological Action of DC and Low-Frequency AC Magnetic Fields. Bioelectromagnetics, 22, 27-45. https://doi.org/10.1002/1521-186X(200101)22:1\%3C27::AID-BEM4\%3E3.0.CO;2-2 https://www.aipro.info/wp/wp-content/uploads/2017/08/Reviewof_Russian_Literat ureon.pdf

[6] Lee, H.C., Hong, M.-N., Jung, S.H., Kim, B.C., Suh, Y.J., Ko, Y.-G., et al. (2015) Effect of Extremely Low Frequency Magnetic Fields on Cell Proliferation and Gene Expression. Bioelectromagnetics, 36, 506-516. https://doi.org/10.1002/bem.21932

[7] Markov, M. (2011) Nonthermal Mechanism of Interactions between Electromagnetic Fields and Biological Systems: A Calmodulin Example. The Environmentalist, 31, 114-120. https://doi.org/10.1007/s10669-011-9321-1

[8] Romanenko, S., Begley, R., Harvey, A.R., Hool, L. and Wallace, V.P. (2017) The Interaction between Electromagnetic Fields at Megahertz, Gigahertz and Terahertz Frequencies With Cells, Tissues and Organisms: Risks and Potential. Journal of the Royal Society Interface, 14, Article ID: 20170585.

https://doi.org/10.1098/rsif.2017.0585

https://www.ncbi.nlm.nih.gov/pmc/articles/PMC5746568/

[9] Bischof, M. and Del Giudice, E. (2013) Communication and the Emergence of Collective Behavior in Living Organisms: A Quantum Approach. Molecular Biology International, 2013, Article ID: 987549. https://doi.org/10.1155/2013/987549 http://www.oalib.com/paper/3079684\#.XJyuG7ieErs

[10] Tigrek, S. and Barnes, F. (2010) Water Structures ad Effects of Electric and Magnetic Fields. European Journal of Oncology, 5, 25-50. http://www.teslabel.be/PDF/ICEMS Monograph 2010.pdf

[11] Enami, S. and Colussi, A.J. (2013) Long-Range Specific Ion-Ion Interactions in Hydrogen-Bonded Liquid Films. The Journal of Chemical Physics, 138, Article ID: 184706. https://doi.org/10.1063/1.4803652 https://pdfs.semanticscholar.org/1917/fadacf2b318da1d31b95c489483dd33ab30d.pdf

[12] Gold, S., Goodman, R. and Shirley-Henderson, A. (1994) Exposure of Simian Vi- 
rus-40-Transformed Human Cells to Magnetic Fields Results in Increased Levels of T-Antigen mRNA and Protein. Bioelectromagnetics, 15, 329-336. https://doi.org/10.1002/bem.2250150407

[13] Nguye, T.H.P., Pham, V.T.H., Nguyen, S.H., Baulin, V., Croft, R.J., Phillips, B., et al. (2016) The Bioeffects Resulting from Prokaryotic Cells and Yeast Being Exposed to an $18 \mathrm{GHz}$ Electromagnetic Field. PLoS ONE, 11, Article ID: e0158135. https://doi.org/10.1371/journal.pone.0158135 https://journals.plos.org/plosone/article?id=10.1371/journal.pone.0158135\#pone.01 58135.ref023

[14] Liboff, A.R. (2010) Weak Low-Frequency Electromagnetic Fields Are Biologically Interactive. European Journal of Oncology, 5, 51-61.

http://www.teslabel.be/PDF/ICEMS Monograph 2010.pdf

[15] Volkenstein, M.K. (1981) Simple Physical Presentation of Enzymatic Catalysis. Journal of Theoretical Biology, 89, 45-51. https://doi.org/10.1016/0022-5193(81)90178-8

[16] Jacobson, J.I. (2016) A Quantum Theory of Disease, Including Cancer and Aging. Integrative Molecular Medicine, 3, 524-541. https://doi.org/10.15761/IMM.1000200 https://www.oatext.com/A-quantum-theory-of-disease-including-cancer-and-aging. php

[17] Srivastava, Y., Sassaroli, E., Swain, J., Widom, A., Narain, M. and de Montmollin, G. (2020) Non-Chemical Signatures of Biological Materials: Radio Signals from Covid19? Electromagnetic Biology and Medicine, 39, 340-346. https://doi.org/10.1080/15368378.2020.1803081 https://www.tandfonline.com/doi/full/10.1080/15368378.2020.1803081? src=recsys

[18] Masters, P.S. (2006) The Molecular Biology of Coronaviruses. Advances in Virus Research, 66, 193-292. https://doi.org/10.1016/S0065-3527(06)66005-3

https://www.ncbi.nlm.nih.gov/pmc/articles/PMC7112330/

[19] Andersen, K.G., Rambaut, A., Lipkin, W.I., Holmes, E.C. and Garry, R.F. (2020) The Proximal Origin of SARS-CoV-2. Nature Medicine, 26, 450-452. https://doi.org/10.1038/s41591-020-0820-9 https://www.nature.com/articles/s41591-020-0820-9

[20] Zhou, P., Yang, X.L., Wang, X.G., Hu, B., Zhang, L., Zhang, W., et al. (2020) A Pneumonia Outbreak Associated with a New Coronavirus of Probable Bat Origin. Nature, 579, 270-273. https://doi.org/10.1038/s41586-020-2012-7

[21] Alsaadi, E.A.J., Jones, I.M. (2019) Membrane Binding Proteins of Coronaviruses. Future Virology, 14, 275-286. https://doi.org/10.2217/fvl-2018-0144 https://www.futuremedicine.com/doi/10.2217/fvl-2018-0144

[22] Almeida, J.D., et al. (1968) Virology: Coronaviruses. Nature, 220, 650. https://doi.org/10.1038/220650b0 https://www.ncbi.nlm.nih.gov/pmc/articles/PMC7086490/pdf/41586 1968 Article BF220650b0.pdf

[23] Bullock, H.A., Goldsmith, C.S., Zaki, S.R., Martines, R.B. and Miller, S.E. (2021) Difficulties in Differentiating Coronaviruses from Subcellular Structures in Human Tissues by Electron Microscopy. Emerging Infectious Diseases, 27, 1023-1031. https://doi.org/10.3201/eid2704.204337 https://www.ncbi.nlm.nih.gov/pmc/articles/PMC8007326/

[24] Macnaughton, M.R. and Davies, H.A. (1981) Enteric Coronaviruses. Archives of Virology, 70, 301-313. https://doi.org/10.1007/BF01320245

https://www.ncbi.nlm.nih.gov/pmc/articles/PMC7087291/pdf/705 2005 Article BF 
01320245.pdf

[25] Caul, E.O., Ashley, C.R. and Egglestone, S.I. (1977) Recognition of Human Enteric Coronaviruses by Electron Microscopy. Medical Laboratory Sciences, 34, 259-263.

[26] Nordzieke, D.E. and Medraño-Fernandez, I. (2018) The Plasma Membrane: A Platform for Intra- and Intercellular Redox. Antioxidants, 7, Article No. 168.

https://doi.org/10.3390/antiox7110168 https://www.mdpi.com/2076-3921/7/11/168

[27] Pawłowski, P.H. (2021) Charged Amino Acids May Promote Coronavirus SARS-CoV-2 Fusion with the Host Cell. AIMS Biophysics, 8, 111-120.

https://doi.org/10.3934/biophy.2021008 http://www.aimspress.com/article/id/600e8564ba35de4aca6cff2f

[28] Yao, Q., Masters, P.S. and Ye, R. (2013) Negatively Charged Residues in the Endodomain Are Critical for Specific Assembly of Spike Protein into Murine Coronavirus. Virology, 442, 74-81. https://doi.org/10.1016/j.virol.2013.04.001 https://www.ncbi.nlm.nih.gov/pmc/articles/PMC3772176/

[29] Chen. X. and Geiger, J.D. (2020) Janus Sword Actions of Chloroquine and Hydroxychloroquine against COVID-19. Cellular Signalling, 73, Article ID: 109706.

https://doi.org/10.1016/j.cellsig.2020.109706 https://www.ncbi.nlm.nih.gov/pmc/articles/PMC7333634/

[30] Kneller, D.W., Phillips, G., Weiss, K.L., Zhang, Q., Coates, L. and Kovalevsky, A. (2021) Direct Observation of Protonation State Modulation in SARS-CoV-2 Main Protease upon Inhibitor Binding with Neutron Crystallography. Journal of Medicinal Chemistry, 64, 4991-5000. https://doi.org/10.1021/acs.jmedchem.1c00058 https://pubs.acs.org/doi/10.1021/acs.jmedchem.1c00058

[31] Warwicker, J. (2021) A Model for pH Coupling of the SARS-CoV-2 Spike Protein Open/Closed Equilibrium. Briefings in Bioinformatics, 22, 1499-1507. https://doi.org/10.1093/bib/bbab056 https://www.ncbi.nlm.nih.gov/pmc/articles/PMC8108619/\#ref6

[32] Xia, X. (2021) Domains and Functions of Spike Protein in SARS-Cov-2 in the Context of Vaccine Design. Viruses, 13, Article No. 109.

https://doi.org/10.3390/v13010109

https://www.ncbi.nlm.nih.gov/pmc/articles/PMC7829931/

[33] Sarkar, M., Etheimer, P. and Saha, S. (2021-Preprint) Viroporin Activity of SARS-CoV-2 Orf3a and Envelope Protein Impacts Viral Pathogenicity. Biorxiv. https://doi.org/10.1101/2021.09.26.461873 https://www.biorxiv.org/content/10.1101/2021.09.26.461873v1

[34] Castaño-Rodriguez, C., Honrubia, J.M., Gutiérrez-Álvarez, J., DeDiego, M.L., Nieto-Torres, J.L., Jimenez-Guardeño, J.M., et al. (2018) Role of Severe Acute Respiratory Syndrome Coronavirus Viroporins E, 3a, and 8a in Replication and Pathogenesis. ASM Journals/mBio, 9, Article ID: e02325-17. https://doi.org/10.1099/vir.0.000201 https://journals.asm.org/doi/10.1128/mBio.02325-17

[35] Scott, C. and Griffin, S. (2015) Viroporins: Structure, Function and Potential as Antiviral Targets. Journal of General Virology, 96, 2000-2027.

https://www.microbiologyresearch.org/docserver/fulltext/jgv/96/8/2000 vir000201. pdf? expires $=1635582918 \&$ id $=i d \& a c c n a m e=$ guest $\&$ checksum $=4$ B90B74DD1AEDB9 9DC61222C435CFC97

[36] Kundrotas, P.J. and Alexov, E. (2006) Electrostatic Properties of Protein-Protein Complexes. Biophysical Journal, 91, 1724-1736. 
https://doi.org/10.1529/biophysj.106.086025 https://www.ncbi.nlm.nih.gov/pmc/articles/PMC1544298/

[37] Zhou, H.X. and Pang, X. (2018) Electrostatic Interactions in Protein Structure, Folding, Binding, and Condensation. Chemical Reviews, 118, 1691-1741.

https://doi.org/10.1021/acs.chemrev.7b00305

https://www.ncbi.nlm.nih.gov/pmc/articles/PMC5831536/

[38] Shashikala, H., Chakravorty, A. and Alexov, E. (2019) Modeling Electrostatic Force in Protein-Protein Recognition. Frontiers in Molecular Biosciences, 6, Article No. 94. https://doi.org/10.3389/fmolb.2019.00094 https://www.ncbi.nlm.nih.gov/pmc/articles/PMC6774301/

[39] Turner, A.J. (2015) ACE2 Cell Biology, Regulation, and Physiological Functions. In: Unger, T., Steckelings, U.M. and dos Santos, R.A.S., (Authors), The Protective Arm of the Renin Angiotensin System (RAS), Academic Press, Cambridge, 185-189.

https://doi.org/10.1016/B978-0-12-801364-9.00025-0 https://www.ncbi.nlm.nih.gov/pmc/articles/PMC7149539/

[40] Luchini, A., Micciulla, S., Corucci, G., Batchu, K.C., Santamaria, A., Laux, V., et al. (2021) Lipid Bilayer Degradation Induced by SARS-CoV-2 Spike Protein as Revealed by Neutron Reflectometry. Scientific Reports, 11, Article No. 14867.

https://www.nature.com/articles/s41598-021-93996-x

https://doi.org/10.1038/s41598-021-93996-X

[41] Suzuki, Y.J. and Gychka, S.G. (2021) SARS-CoV-2 Spike Protein Elicits Cell Signaling in Human Host Cells: Implications for Possible Consequences of COVID-19 Vaccines. Vaccines, 9, Article No. 36. https://doi.org/10.3390/vaccines9010036 https://www.ncbi.nlm.nih.gov/pmc/articles/PMC7827936/

[42] Gorshkov, K., Susumu, K., Chen, J., Xu, M., Pradhan, M., Zhu, W., et al. (2020) Quantum Dot-Conjugated SARS-CoV-2 Spike Pseudo-Virions Enable Tracking of Angiotensin Converting Enzyme 2 Binding and Endocytosis. ACS Nano, 14, 12234-12247. https://doi.org/10.1021/acsnano.0c05975

[43] Du, E., Qiang, Y. and Liu, J. (2018) Erythrocyte Membrane Failure by Electromechanical Stress. Applied Sciences, 8, Article No. 174.

https://doi.org/10.3390/app8020174 https://www.ncbi.nlm.nih.gov/pmc/articles/PMC5909407/

[44] Lande, M.B., Donovan, J.M. and Zeidel, M.L. (1995) The Relationship between Membrane Fluidity and Permeabilities to Water, Solutes, Ammonia, and Protons. Journal of General Physiology, 106, 67-84. https://doi.org/10.1085/igp.106.1.67

[45] Shimanouchi, T., Ishii, H., Yoshimoto, N., Umakoshi, H. and Kuboi, R. (2009) Calcein Permeation across Phosphatidylcholine bilayer Membrane: Effects of Membrane Fluidity, Liposome Size, and Immobilization. Colloids and Surfaces B: Biointerfaces, 73, 156-160. https://doi.org/10.1016/j.colsurfb.2009.05.014

[46] Watanabe, Y., Bowden, T.A., Wilson, I.A. and Crispin, M. (2019) Exploitation of Glycosylation in Enveloped Virus Pathobiology. Biochimica et Biophysica Acta General Subjects, 1863, 1480-1497. https://doi.org/10.1016/j.bbagen.2019.05.012 https://www.ncbi.nlm.nih.gov/pmc/articles/PMC6686077/

[47] Zhao, Z., Chen, H. and Wang, H. (2021) Glycans of SARS-CoV-2 Spike Protein in Virus Infection and Antibody Production. Frontiers in Molecular Biosciences, 8, Article No. 629873. https://doi.org/10.3389/fmolb.2021.629873 https://www.frontiersin.org/articles/10.3389/fmolb.2021.629873/full

[48] Correa, Y., Waldie, S., Thépaut, M., Micciulla, S., Moulin, M., Fieschi, F., et al. (2021) SARS-CoV-2 Spike Protein Removes Lipids from Model Membranes and 
Interferes with the Capacity of High Density Lipoprotein to Exchange Lipids. Journal of Colloid and Interface Science, 602, 732-739.

https://doi.org/10.1016/j.jcis.2021.06.056

https://www.ncbi.nlm.nih.gov/pmc/articles/PMC8195693/

[49] Lai, A.L. and Freed, J.H. (2021) SARS-CoV-2 Fusion Peptide has a Greater Membrane Perturbating Effect than SARS-CoV with Highly Specific Dependence on $\mathrm{Ca}^{2+}$. Journal of Molecular Biology, 433, Article ID: 166946.

https://doi.org/10.1016/j.jmb.2021.166946

https://www.ncbi.nlm.nih.gov/pmc/articles/PMC7969826/

[50] Robert, D., Lauritzen, A. and Seneff, S. (2013) Biological Water Dynamics and Entropy: A Biophysical Origin of Cancer and Other Diseases. Entropy, 15, 3822-3876. https://doi.org/10.3390/e15093822

[51] Verdiá-Báguena, C., Nieto-Torres, J.L., Alcaraz, A., DeDiego, M.L., Torres, J., Aguilella, V.M., et al. (2012) Coronavirus E Protein forms Ion Channels with Functionally and Structurally-Involved Membrane Lipids. Virology, 432, 485-494.

https://doi.org/10.1016/j.virol.2012.07.005

https://www.ncbi.nlm.nih.gov/pmc/articles/PMC3438407/

[52] McClenaghan, C., Hanson, A., Lee, S.J. and Nichols, C.G. (2020) Coronavirus Proteins as Ion Channels: Current and Potential Research. Frontiers in Immunology, 11, Article No. 573339. https://doi.org/10.3389/fimmu.2020.573339 https://www.frontiersin.org/articles/10.3389/fimmu.2020.573339/full

[53] Yu, M., Zhang, T., Zhang, W., Sun, Q., Li, H. and Li, J.P. (2021) Elucidating the Interactions Between Heparin/Heparan Sulfate and SARS-CoV-2-Related Proteins-An Important Strategy for Developing Novel Therapeutics for the COVID-19 Pandemic. Frontiers in Molecular Biosciences, 7, Article No. 628551.

https://doi.org/10.3389/fmolb.2020.628551

https://www.frontiersin.org/articles/10.3389/fmolb.2020.628551/full

[54] Yue, J., Jin, W., Yang, H., Faulkner, J., Song, X., Qiu, H., et al. (2021) Heparan Sulfate Facilitates Spike Protein-Mediated SARS-CoV-2 Host Cell Invasion and Contributes to Increased Infection of SARS-CoV-2 G614 Mutant and in Lung Cancer. Frontiers in Molecular Biosciences, 8, Article No. 649575.

https://doi.org/10.3389/fmolb.2021.649575

https://www.frontiersin.org/articles/10.3389/fmolb.2021.649575/full

[55] Pincemail, J., Cavalier, E., Charlier, C., Cheramy-Bien, J.P., Brevers, E., Courtois, A., et al. (2021) Oxidative Stress Status in COVID-19 Patients Hospitalized in Intensive Care Unit for Severe Pneumonia. A Pilot Study. Antioxidants, 10, Article No. 257. https://doi.org/10.3390/antiox10020257

[56] Pokorný, J., Pokorný, J., Foletti, A., Kobilková, J., Vrba, J. and Vrba, J. (2015) Mitochondrial Dysfunction and Disturbed Coherence: Gate to Cancer. Pharmaceuticals, 8, 675-695. https://doi.org/10.3390/ph8040675 https://www.ncbi.nlm.nih.gov/pmc/articles/PMC4695805/

[57] Thar, R. and Kühl, M. (2004) Propagation of Electromagnetic Radiation in Mitochondria? Journal of Theoretical Biology, 230, 261-270.

https://doi.org/10.1016/j.jtbi.2004.05.021

https://www.researchgate.net/publication/8407688 Propagation of electromagneti c radiation in mitochondria

[58] Pokorný, J., Pokorný, J. and Borodavka, F. (2017) Warburg Effect-Damping of Electromagnetic Oscillations. Electromagnetic Biology and Medicine, 36, 270-278. https://doi.org/10.1080/15368378.2017.1326933 https://emmind.net/openpapers_repos/Endogenous_Fields-Mind/General/EM Can 
cer/2017 Warburg effect damping of electromagnetic oscillations.pdf

[59] Pokorný, J. (2014) Cancer-Pathological Breakdown of Coherent Energy States. Biophysical Reviews and Letters, 9, 115-133.

https://doi.org/10.1142/S1793048013300077

https://www.researchgate.net/profile/Jan Vrba/publication/263805231 CANCER-P Athologi-

cal breakdown of coherent energy states/links/57207e6308aed056fa236c4d.pdf?di $\underline{\text { sableCoverPage }=\text { true }}$

[60] Petersen, R.C., Reddy, M.S. and Liu, P-R. (2018) Advancements in Free-Radical Pathologies and an Important Treatment Solution with a Free-Radical Inhibitor. Science Forecast Journal of Biotechnology and Biomedical Engineering, 1, Article No. 1003. https://scienceforecastoa.com/Articles/SJBBE-V1-E1-1003.pdf

[61] Yang, M. and Lai, C.L. (2020) SARS-CoV-2 Infection: Can Ferroptosis Be a Potential Treatment Target for Multiple Organ Involvement? Cellular Death Discovery, 6, Article No. 130. https://doi.org/10.1038/s41420-020-00369-w https://www.nature.com/articles/s41420-020-00369-w

[62] Cecchini, R. and Cecchini, A.L. (2020) SARS-CoV-2 Infection Pathogenesis Is Related to Oxidative Stress as a Response to Aggression. Medical Hypotheses, 143, Article ID: 110102. https://doi.org/10.1016/j.mehy.2020.110102 https://www.ncbi.nlm.nih.gov/pmc/articles/PMC7357498/

[63] Majumdar, S. and Murphy, P.M. (2021) Chemokine Regulation during Epidemic Coronavirus Infection. Frontiers in Pharmacology, 11, Article No. 600369. https://doi.org/10.3389/fphar.2020.600369 https://www.frontiersin.org/articles/10.3389/fphar.2020.600369/full

[64] Georgiou, C.D. (2010) Oxidative Stress-Induced Biological Damage by Low-Level EMFs: Mechanism of Free Radical Pair Electron Spin-Polarization and Biochemical Amplification. European Journal of Oncology, 5, 63-113. http://www.teslabel.be/PDF/ICEMS Monograph 2010.pdf

[65] Robert, D., Lauritzen, A. and Seneff, S. (2013) Biological Water Dynamics and Entropy: A Biophysical Origin of Cancer and Other Diseases. Entropy, 15, 3822-3876. https://doi.org/10.3390/e15093822 https://people.csail.mit.edu/seneff/Entropy/entropy-15-03822.pdf

[66] Sendner, C., Horinek, D., Bocquet, L. and Netz, R.R. (2009) Interfacial Water at Hydrophobic and Hydrophilic Surfaces: Slip, Viscosity, and Diffusion. Langmuir, 25, 10768-10781. https://doi.org/10.1021/la901314b https://www.academia.edu/16943925/Interfacial Water at Hydrophobic and Hyd rophilic Surfaces Slip Viscosity and Diffusion

[67] Patel, A.J., Varilly, P., Jamadagni, S.N., Hagan, M.F., Chandler, D. and Garde, S. (2012) Sitting at the Edge: How Biomolecules Use Hydrophobicity to Tune Their Interactions and Function. The Journal of Physical Chemistry B, 116, 2498-2503. https://doi.org/10.1021/jp2107523 https://www.ncbi.nlm.nih.gov/pmc/articles/PMC3303187/

[68] Antonenko, Y.N., Pohl, P. and Rosenfeld, E. (1996) Visualization of the Reaction Layer in the Immediate Membrane Vicinity. Archives of Biochemistry and Biophysics, 333, 225-232. https://doi.org/10.1006/abbi.1996.0385

[69] Pollack, G.H. (2013) The Fourth Phase of Water: Beyond Solid, Liquid, and Vapor. Edgescience, 16, 14-18.

[70] Messori, C. (2019) Deep into the Water: Exploring the Hydro-Electromagnetic and Quantum-Electrodynamic Properties of Interfacial Water in Living Systems. Open 
Access Library Journal, 6, 1-5. https://doi.org/10.4236/oalib.1105435

[71] Messori, C. (2019) The Super-Coherent State of Biological Water. Open Access Library Journal, 6, 1-17. https://doi.org/10.4236/oalib.1105236 https://www.scirp.org/journal/PaperInformation.aspx?PaperID=90862

[72] Preparata, G., Messina, B., Talpo, G., Ettore Gigante, G., Del Giudice, E., Bizzarri, M., et al. (1999) The Role of QED (Quantum Electro Dynamics) in Medicine. Rivista di Biologial Biology Forum 93/2000, 1-27. http://www.22passi.it/downloads/biorisonanza/qeddefinitivo.pdf

[73] Pollack, G.H., Figueroa, X. and Zhao, Q. (2009) Molecules, Water, and Radiant Energy: New Clues for the Origin of Life. International Journal of Molecular Sciences, 10, 1419-1429. https://doi.org/10.3390/ijms10041419 https://www.ncbi.nlm.nih.gov/pmc/articles/PMC2680624/

[74] Hwang, S.G., Hong, J.K., Sharma, A., Pollack, G.H. and Bahng, G. (2018) Exclusion Zone and Heterogeneous Water Structure at Ambient Temperature. PLoS ONE, 13, Article ID: e0195057. https://doi.org/10.1371/journal.pone.0195057 https://journals.plos.org/plosone/article?id=10.1371/journal.pone.0195057

[75] Del Giudice, E., Spinetti, P.R. and Tedeschi, A. (2010) Water Dynamics at the Root of Metamorphosis in Living Organisms. Water, 2, 566-586.

https://doi.org/10.3390/w2030566 https://pdfs.semanticscholar.org/4bc0/3fdbd99780f6713d375a74bd9200d525b2a7.p df

[76] Del Giudice, E., Tedeschi, A., Vitiello, G. and Voeikov, V. (2013) Coherent Structures in Liquid Water close to Hydrophilic Surfaces. Journal of Physics. Conference Series, 442, Article ID: 012028. https://doi.org/10.1088/1742-6596/442/1/012028 https://iopscience.iop.org/article/10.1088/1742-6596/442/1/012028/pdf

[77] Madl, P., De Filippis, A. and Tedeschi, A. (2020) Effects of Ultra-Weak Fractal Electromagnetic Signals on the Aqueous Phase in Living Systems: A Test-Case Analysis of Molecular Rejuvenation Markers in Fibroblasts. Electromagnetic Biology and Medicine, 39, 227-238. https://doi.org/10.1080/15368378.2020.1762634 https://www.tandfonline.com/doi/full/10.1080/15368378.2020.1762634

[78] Del Giudice, E., Pulselli, R.M. and Tiezzi, E. (2009) Thermodynamics of Irreversible Processes and Quantum Field Theory: An Interplay for the Understanding of Ecosystem Dynamics. Ecological Modelling, 220, 1874-1879.

https://doi.org/10.1016/j.ecolmodel.2009.04.035

[79] Brizhik, L., Del Giudice, E., Jørgensen, S.E., Marchettini, N. and Tiezzi, E. (2009) The Role of Electromagnetic Potentials in the Evolutionary Dynamics of Ecosystems. Ecological Modelling, 220, 1865-1869.

https://doi.org/10.1016/j.ecolmodel.2009.04.017 https://www.researchgate.net/publication/222525342 The role of electromagnetic potentials in the evolutionary dynamics of ecosystems

[80] Brizhik, L., Del Giudice, E., Tedeschi, A. and Voeikov, V.L. (2011) The Role of Water in the Information Exchange between the Components of an Ecosystem. Ecological Modelling, 222, 2869-2877. https://doi.org/10.1016/j.ecolmodel.2011.05.017 https://www.researchgate.net/publication/229327908 The role of water in the in formation exchange between the components of an ecosystem

[81] Brizhik, L., Chiappini, E., Stefanini, P. and Vitiello, G. (2018) Modeling Meridians within the Quantum Field Theory. Journal of Acupuncture and Meridian Studies, 12, 29-36. https://doi.org/10.1016/j.jams.2018.06.009

[82] Marchettini, N., Del Giudice, E., Voeikov, V. and Tiezzi, E. (2010) Water: A Me- 
dium Where Dissipative Structures Are Produced by a Coherent Dynamics. Journal of Theoretical Biology, 265, 511-516. https://doi.org/10.1016/j.jtbi.2010.05.021

[83] Adamski, A.G. (2011) Bioplasma Concept of Consciousness. NeuroQuantology, 9, 681-691.

http://citeseerx.ist.psu.edu/viewdoc/download?doi=10.1.1.841.4586\&rep=rep1\&type $=$ pdf

[84] Szent-Gyorgyi, A. (1957) Bioenergetics. Academic Press, New York

[85] Cristof Grewer, A.G., Thomas, M. and Klaus, F. (2013) Electrophysiological Characterization of Membrane Transport Proteins. Annual Review of Biophysics, 42, 95-120. https://doi.org/10.1146/annurev-biophys-083012-130312

[86] Watanabe, R., Soga, N., Fujita, D., Tabata, K.V., Yamauchi, L., Hyeon Kim, S., et al. (2014) Arrayed Lipid Bilayer Chambers Allow Single-Molecule Analysis of Membrane Transporter Activity. Nature Communications, 5, Article No. 4519.

https://doi.org/10.1038/ncomms5519

https://www.nature.com/articles/ncomms5519

[87] Gutiérrez-Sanz, Ó., Tapia, C., Marques, M.C., Zacarias, S., Vélez, M., Pereira, I.A., et al. (2015) Induction of a Proton Gradient across a Gold-Supported Biomimetic Membrane by Electroenzymatic $\mathrm{H}_{2}$ Oxidation. Angewandte Chemie International Edition, 54, 2684-2687. https://doi.org/10.1002/anie.201411182

[88] Decoursey, T.E. (2003) Voltage-Gated Proton Channels and other Proton Transfer Pathways. Physiological Reviews, 83, 475-579.

https://doi.org/10.1152/physrev.00028.2002

[89] Pomès, R. and Roux, B. (1998) Free Energy Profiles for $\mathrm{H}^{+}$Conduction along Hydrogen-Bonded Chains of Water Molecules. Biophysical Journal, 75, 33-40.

https://doi.org/10.1016/S0006-3495(98)77492-2 https://www.ncbi.nlm.nih.gov/pmc/articles/PMC1299677/

[90] Hassanali, A., Giberti, F., Cuny, J., Kühne, T.D. and Parrinello, M. (2013) Proton Transfer Through the Water Grossamer. Proceedings of the National Academy of Sciences of the United States of America, 110, 13723-13728. https://doi.org/10.1073/pnas.1306642110

[91] Peng, Y., Swanson, J.M., Kang, S.G., Zhou, R., Voth, G.A., et al. (2015) Hydrated Excess Protons Can Create their Own Water Wires. Journal of Physical Chemistry $B, 119,9212-9218$. https://doi.org/10.1021/jp5095118 https://pubs.acs.org/doi/10.1021/jp5095118

[92] Mather, J.P. and Roberts, P.E. (1998) Introduction to Cell and Tissue Culture Theory and Technique. Plenum Press, New York.

https://www.bjcancer.org/Sites OldFiles/ Library/UserFiles/pdf/Introduction\%20to \%20Cell\%20and\%20Tissue\%20Culture.pdf

[93] Ullmann, G.M. and Bombarda, E. (2013) $\mathrm{p} K_{a}$ Values and Redox Potentials of Proteins. What Do They Mean? Biological Chemistry, 394, 611-619.

http://www.bisb.uni-bayreuth.de/PDF/Ullmann2013.BiolChem.pdf

[94] Darcy, J.W.W., Koronkiewicz, B., Parada, G.A. and Mayer, J.M. (2018) A Continuum of Proton-Coupled Electron Transfer Reactivity. Accounts of Chemical Research, 51, 2391-2399. https://doi.org/10.1021/acs.accounts.8b00319

http://gaznevada.iq.usp.br/wp-content/uploads/2018/10/mayer-18 PCET continuu $\underline{\text { m.pdf }}$

[95] Huynh, M.H. and Meyer, T.J. (2007) Proton-Coupled Electron Transfer. Chemical Reviews, 107, 5004-5064. https://doi.org/10.1021/cr0500030 https://www.ncbi.nlm.nih.gov/pmc/articles/PMC3449329/ 
[96] Rohani, M. and Pollack, G.H. (2013) Flow through Horizontal Tubes Submerged in Water in the Absence of a Pressure Gradient: Mechanistic Considerations. Langmuir, 29, 6556-6561. https://doi.org/10.1021/la4001945

[97] Riddick, T.M. (1968) Control of Colloid Stability through Zeta Potential. With a Closing Chapter on Its Relationship to Cardiovascular Disease. Published for Zeta Meter by Livingston Publishing Company, Pennsylvania.

[98] Pollack, G.H. (2017) Why Hydrogels Don't Dribble Water. Gels, 3, Article No. 43. https://doi.org/10.3390/gels3040043 https://www.mdpi.com/2310-2861/3/4/43/pdf

[99] Yu, A., Carlson, P. and Pollack, G.H (2014) Unexpected Axial Flow through Hydrophilic Tubes: Implications for Energetics of Water. The European Physical Journal Special Topics, 223, 947-958. https://doi.org/10.1140/epjst/e2013-01837-8

[100] Qiana, S. and Bau, H.H. (2009) Magneto-Hydrodynamics Based Microfluidics. Mechanics Research Communications, 36, 10-21. https://doi.org/10.1016/j.mechrescom.2008.06.013 https://www.ncbi.nlm.nih.gov/pmc/articles/PMC2768299/

[101] Hanson, A. (2021) Spontaneous Electrical Low-Frequency Oscillations: A Possible Role in Hydra and All Living Systems. Philosophical Transactions of the Royal Society B, 376, Article ID: 20190763. https://doi.org/10.1098/rstb.2019.0763 https://royalsocietypublishing.org/doi/10.1098/rstb.2019.0763

[102] Del Giudice, E., Fleischmann, M., Preparata, G. and Talpo, G. (2002) On the "Unreasonable" Effects of ELF Magnetic Fields upon a System of Ions. Bioelectromagnetics, 23, 522-530. https://doi.org/10.1002/bem.10046

[103] Tessaro, L., Dotta, B.T. and Persinger, M.A. (2019) Bacterial Biophotons as Non-Local Information Carriers: Species-Specific Spectral Characteristics of a Stress Response. MicrobiologyOpen, 8, Article No. e00761. https://doi.org/10.1002/mbo3.761 https://www.ncbi.nlm.nih.gov/pmc/articles/PMC6562132/

[104] Blackman, C.F., Blanchard, J.P., Benane, S.G. and House, D.E. (1995) The Ion Parametric Resonance Model Predicts Magnetic Field Parameters That Affect Nerve Cells. The FASEB Journal, 9, 547-551. https://doi.org/10.1096/fasebj.9.7.7737464 https://www.researchgate.net/publication/215658509 The ion parametric resonan ce model predicts magnetic field parameters that affect nerve cells

[105] Giuliani, L., D’Emilia, E., Grimaldi, S., Lisi, A., Bobkova, N. and Zhadin, M.N. (2009) Investigating the Icr Effect in a Zhadin's Cell. International Journal of Biomedical Science, 5, 181-186.

https://www.ncbi.nlm.nih.gov/pmc/articles/PMC3614765/

[106] Montagnier, L., Aïssa, J., Ferris, S., Montagnier, J.L. and Lavallée, C. (2009) Electromagnetic Signals Are Produced by Aqueous Nanostructures Derived from Bacterial DNA Sequences. Interdisciplinary Sciences Computational Life Sciences, 1, 81-90. https://doi.org/10.1007/s12539-009-0036-7

http://sphq.org/wp-content/uploads/2016/03/etudes Montagnier Electro-signals-p roduced-by-aqueous-DNA.pdf

[107] Friedman, R. (2018) Membrane-Ion Interactions. The Journal of Membrane Biology, 251, 453-460. https://doi.org/10.1007/s00232-017-0010-y https://www.ncbi.nlm.nih.gov/pmc/articles/PMC6028871/

[108] Montagnier, L., Del Giudice, E., Aïssa, J., Lavallee, C., Motschwiller, S., Capolupo, A., et al. (2015) Transduction of DNA Information through Water and Electromagnetic Waves. Electromagnetic Biology and Medicine, 34, 106-112. https://doi.org/10.3109/15368378.2015.1036072 
https://arxiv.org/abs/1501.01620

[109] Radil, R., Barabas, J., Janousek, L. and Bereta, M. (2020) Frequency Dependent Alterations of $S$. Cerevisiae Proliferation Due to LF EMF Exposure. Advances in Electrical and Electronic Engineering, 18, 99-106.

https://doi.org/10.15598/aeee.v18i2.3461

http://advances.utc.sk/index.php/AEEE/article/view/3461

[110] Liboff, A.R. (2019) ION Cyclotron Resonance: Geomagnetic Strategy for Living Systems? Electromagnetic Biology and Medicine, 38, 143-148. https://doi.org/10.1080/15368378.2019.1608234

[111] Zhadin, M.N., Novikov, V.V., Barnes, F.S. and Pergola, N.F. (1998) Combined Action of Static and Alternating Magnetic Fields on Ionic Current in Aqueous Glutamic Acid Solution. Bioelectromagnetics, 19, 41-45.

https://doi.org/10.1002/(SICI)1521-186X(1998)19:1\%3C41::AID-BEM4\%3E3.0.CO; $\underline{2-4}$

[112] Comisso, N., Del Giudice, E., De Ninno, A., Fleischmann, M., Giuliani, L., Mengoli, G., et al. (2006) Dynamics of the Ion Cyclotron Resonance Effect on Amino Acids Adsorbed at the Interfaces. Bioelectromagnetics, 27, 16-25.

https://doi.org/10.1002/bem.20171

[113] Widom, A., Swain, J. and Valenci, V.I. (2021) Extremely Low Frequency Ion Cyclotron Resonances on the Surface Boundaries of Coherent Water Domains. IOP Conference Series. Earth and Environmental Science, 853, Article ID: 012024. https://doi.org/10.1088/1755-1315/853/1/012024 https://iopscience.iop.org/article/10.1088/1755-1315/853/1/012024/pdf

[114] Binhi, V.N. (1998) Interference Mechanism for Some Biological Effects of Pulsed Magnetic Fields. Bioelectrochemistry and Bioenergetics, 45, 73-81.

https://doi.org/10.1016/S0302-4598(98)00078-6

https://citeseerx.ist.psu.edu/viewdoc/download?doi=10.1.1.1041.4184\&rep=rep1\&ty pe $=$ pdf

[115] Kapri-Pardes, E., Hanoch, T., Maik-Rachline, G., Murbach, M., Bounds, P.L., Kuster, N., et al. (2017) Activation of Signaling Cascades by Weak Extremely Low Frequency Electromagnetic Fields. Cellular Physiology and Biochemistry, 43, 1533-1546. https://doi.org/10.1159/000481977

https://www.karger.com/Article/FullText/481977

[116] Preoteasa, E.A. and Apostol, M.V. (2008) Collective Dynamics of Water in the Living Cell and in Bulk Liquid. New Physical Models and Biological Inferences. International Fröhlich Symposium Biophysical Aspects of Cancer Electromagnetic Mechanisms, Prague. https://arxiv.org/ftp/arxiv/papers/0812/0812.0275.pdf

[117] Davidson, R.M. and Seneff, S. (2012) The Initial Common Pathway of Inflammation, Disease, and Sudden Death. Entropy, 14, 1399-1442.

https://doi.org/10.3390/e14081399

https://dspace.mit.edu/openaccess-disseminate/1721.1/76366

[118] Grasis, J.A. (2017) The Intra-Dependence of Viruses and the Holobiont. Frontiers in Immunology, 8, Article No. 1501. https://doi.org/10.3389/fimmu.2017.01501 https://www.frontiersin.org/articles/10.3389/fimmu.2017.01501/full

[119] Roossinck, M.J. (2015) Move over, Bacteria! Viruses Make Their Mark as Mutualistic Microbial Symbionts. Journal of Virology, 89, 6532-6535.

https://doi.org/10.1128/JVI.02974-14

https://journals.asm.org/doi/10.1128/JVI.02974-14 